\title{
QUASISTATIC CRACK EVOLUTION FOR A COHESIVE ZONE MODEL WITH DIFFERENT RESPONSE TO LOADING AND UNLOADING: A YOUNG MEASURES APPROACH
}

\author{
Filippo Cagnetti ${ }^{1}$ And Rodica TOAdeR $^{2}$
}

\begin{abstract}
A new approach to irreversible quasistatic fracture growth is given, by means of Young measures. The study concerns a cohesive zone model with prescribed crack path, when the material gives different responses to loading and unloading phases. In the particular situation of constant unloading response, the result contained in [G. Dal Maso and C. Zanini, Proc. Roy. Soc. Edinburgh Sect. A 137 (2007) 253-279] is recovered. In this case, the convergence of the discrete time approximations is improved.
\end{abstract}

Mathematics Subject Classification. 49K10, 49Q20.

Received October 8, 2008.

Published online October 9, 2009.

\section{INTRODUCTION}

In this paper we study the fracture growth in an elastic body, taking into account the contribution of the cohesive forces acting between the lips of the crack. We consider materials which display different stressstrain relations in loading and unloading. To focus on this aspect, we keep the rest of the model as simple as possible.

We restrict our analysis to the case of generalized antiplanar shear. More precisely, let $\Omega$ be a bounded open set in $\mathbb{R}^{N}$, with Lipschitz boundary. We assume that the reference configuration is the infinite cylinder $\Omega \times \mathbb{R}$, and that the displacement $U: \Omega \times \mathbb{R} \rightarrow \mathbb{R}^{N+1}$ has the special form $U\left(x_{1}, \ldots, x_{N}, x_{N+1}\right)=\left(0, \ldots, 0, u\left(x_{1}, \ldots, x_{N}\right)\right)$, with $u: \Omega \rightarrow \mathbb{R}$. We assume also that the crack path in the reference configuration is contained in $(\Gamma \cap \bar{\Omega}) \times \mathbb{R}$, where $\Gamma \subset \mathbb{R}^{N}$ is a Lipschitz closed set such that $0<\mathcal{H}^{N-1}(\Gamma \cap \bar{\Omega})<+\infty$ and $\Omega \backslash \Gamma=\Omega^{+} \cup \Omega^{-}$, with $\Omega^{ \pm}$ disjoint open connected sets with Lipschitz boundary. When speaking about bulk and surface energy, we will refer to a finite portion of the cylinder, obtained by intersection with two horizontal hyperplanes separated by a unit distance. Although the case of a planar set $\Omega$ is the most interesting from the point of view of applications, no further relevant technicalities arise in considering an arbitrary $N \geq 2$.

Let us fix a time interval $[0, T]$, with $T>0$. In the situation we consider, the evolution is driven by a time dependent displacement $w:[0, T] \rightarrow H^{1}(\Omega)$ imposed on a fixed portion $\partial_{D} \Omega$ of the boundary $\partial \Omega$. We assume

\footnotetext{
Keywords and phrases. Variational models, energy minimization, free discontinuity problems, crack propagation, Young measures, quasistatic evolution, rate-independent processes.

${ }^{1}$ SISSA, via Beirut 2, 34014 Trieste, Italy. cagnetti@sissa.it

${ }^{2}$ Univ. Udine, Dip. Ingegneria Civile, via delle Scienze 208, 33100 Udine, Italy. toader@uniud.it
} 
that $\partial_{D} \Omega$ is well-separated from $\Gamma$ and that its intersections with $\partial \Omega^{+}$and $\partial \Omega^{-}$have positive $(N-1)$-dimensional measure.

Let us now introduce the energy functional. We suppose that the unbroken part of $\Omega$ can be described in the context of linearized elasticity, so that the stored elastic energy associated to a displacement $u \in H^{1}(\Omega \backslash \Gamma)$ is:

$$
\frac{1}{2} \int_{\Omega \backslash \Gamma}|\nabla u|^{2} \mathrm{~d} x
$$

In order to express the work spent to create a fracture, we need some preliminary notations. Let $u^{ \pm}$denote the trace on $\Gamma$ of the restriction of $u$ to $\Omega^{ \pm}$, and let $[u]$ denote the jump $u^{+}-u^{-}$of $u$ across $\Gamma$. The crack is represented by the set

$$
J_{u}:=\{x \in \Gamma:[u](x) \neq 0\}
$$

Its contribution to the energy, according to Barenblatt's cohesive zone model (see [1]), can be written as

$$
\int_{\Gamma} g(|[u]|) \mathrm{d} \mathcal{H}^{N-1}
$$

where $g:[0,+\infty) \rightarrow[0,+\infty)$ is a $C^{1}$, nondecreasing, bounded, concave function with $g(0)=0$ and $\sigma:=$ $g^{\prime}\left(0^{+}\right) \in(0,+\infty)$. Here $g(|[u]|)$ is the energy per unit area spent to create a crack with opening $|[u]|$. Moreover, $g^{\prime}(|[u]|)$ gives the force per unit area acting between the lips of the crack whose displacements are $u^{+}$and $u^{-}$, respectively. Typically, this force decreases with the distance and hence $g$ is concave. Since in practice the cohesive interactions have finite range, we assume $g$ to be bounded. Therefore, the total energy associated to a displacement $u \in H^{1}(\Omega \backslash \Gamma)$ is given by

$$
E(u):=\frac{1}{2} \int_{\Omega \backslash \Gamma}|\nabla u|^{2} \mathrm{~d} x+\int_{\Gamma} g(|[u]|) \mathrm{d} \mathcal{H}^{N-1} .
$$

Up to now, we did not take into account the dissipation due to the fracturing process. Indeed, it may happen that the imposed boundary data depend on time in such a way that the crack opening first increases (loading phase) and then decreases (unloading phase). In many situations, the energy spent during the loading phase might not be totally recovered during the unloading phase.

In order to describe this phenomenon, we introduce an internal variable $\gamma:[0, T] \rightarrow L^{\infty}(\Gamma)$. For every $x \in \Gamma$ and $t \in[0, T], \gamma(t)(x)$ represents the maximum value reached by the opening of the fracture $|[u](x)|$ at $x$ in the time interval $[0, t]$. Let now $x \in \Gamma$ and $t \in[0, T]$ be fixed. We consider a family of nondecreasing convex functions $\{\varphi(\cdot, z)\}_{z>0}$, each of them defined in $[0, z]$, with the properties: $\varphi(\cdot, z) \geq g(\cdot), \varphi(z, z)=g(z)$, and $\varphi$ is nondecreasing in the second variable. We assume that at time $t$ the energy per unit area of the fracture at the point $x$ is given by $\varphi(|[u](x)|, \gamma(t)(x))$. This means that when $|[u](x)|$ is smaller than the maximal opening reached up to time $t$ the energy density follows a curve that is above $g$. Otherwise, the energy density we consider is still given by the function $g$ (see Fig. 1).

These considerations lead us to describe the state of the system by a pair $(u, \gamma)$ with $|[u]| \leq \gamma$, where $u \in H^{1}(\Omega \backslash \Gamma)$ represents the displacement and $\gamma \in L^{\infty}(\Gamma)$ is the aforementioned internal variable. Thus, we can correct expression (1.1), and the total energy associated to an admissible pair $(u, \gamma)$ is

$$
E(u, \gamma):=\frac{1}{2} \int_{\Omega \backslash \Gamma}|\nabla u|^{2} \mathrm{~d} x+\int_{\Gamma} \varphi(|[u]|, \gamma) \mathrm{d} \mathcal{H}^{N-1}
$$

In order to impose the irreversibility condition, we assume that the variable $\gamma$ is increasing. 


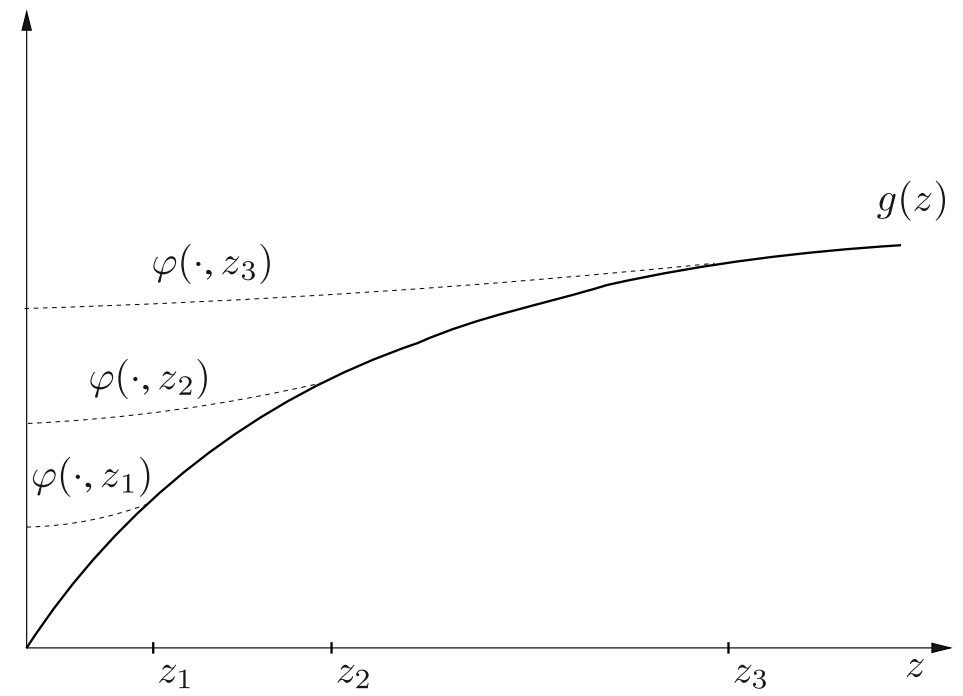

FiguRE 1. Fracture energy per unit surface.

We are now in a position to state the notion of evolution we are interested in. An irreversible quasistatic evolution is a function $t \mapsto(u(t), \gamma(t))$ from $[0, T]$ to $H^{1}(\Omega \backslash \Gamma) \times L^{\infty}(\Gamma)$ such that $u(t)=w(t)$ on $\partial_{D} \Omega$, $|[u(t)]| \leq \gamma(t) \mathcal{H}^{N-1}$-a.e. on $\Gamma$ for every $t \in[0, T]$, and the following conditions are satisfied:

(a) (unilateral) global stability: for every $t \in[0, T]$

$$
E(u(t), \gamma(t)) \leq E(v, \tau)
$$

for every pair $(v, \tau) \in H^{1}(\Omega \backslash \Gamma) \times L^{\infty}(\Gamma)$ such that $v=w(t)$ on $\partial_{D} \Omega$ and $\tau \geq \gamma(t)$;

(b) irreversibility: $t \mapsto \gamma(t)$ is nondecreasing;

(c) energy balance: the function $t \mapsto \int_{\Omega \backslash \Gamma} \nabla u(t) \cdot \nabla \dot{w}(t) \mathrm{d} x$ belongs to $L^{1}([0, T])$ and

$$
E(u(t), \gamma(t))=E(u(0), \gamma(0))+\int_{0}^{t} \int_{\Omega \backslash \Gamma} \nabla u(s) \cdot \nabla \dot{w}(s) \mathrm{d} x \mathrm{~d} s
$$

for every $t \in[0, T]$.

This definition fits into the framework of Mielke's approach to a variational theory of rate independent processes (see [8] and the references therein). Indeed, let us define the stored energy as

$$
\tilde{E}(t,(u, \gamma)):=E(u, \gamma)-\int_{\Gamma} \varphi(0, \gamma) \mathrm{d} \mathcal{H}^{N-1}
$$

if $u=w(t)$ on $\partial_{D} \Omega$ with $|[u]| \leq \gamma \mathcal{H}^{N-1}$-a.e. on $\Gamma$, and the dissipation distance between two admissible pairs $(u, \gamma)$ and $(v, \tau)$ as

$$
\mathcal{D}((u, \gamma),(v, \tau)):=\int_{\Gamma}(\varphi(0, \tau)-\varphi(0, \gamma)) \mathrm{d} \mathcal{H}^{N-1}
$$

if $\tau \geq \gamma \mathcal{H}^{N-1}$-a.e. on $\Gamma$ and $\mathcal{D}((u, \gamma),(v, \tau)):=+\infty$ otherwise. Then,

$$
\partial_{t} \tilde{E}(t,(u, \gamma))=\int_{\Omega \backslash \Gamma} \nabla u \cdot \nabla \dot{w}(t) \mathrm{d} x
$$


and conditions (a)-(c) can be written as

(a) (unilateral) global stability: for every $t \in[0, T]$

$$
\tilde{E}(t,(u(t), \gamma(t))) \leq \tilde{E}(t,(v, \tau))+\mathcal{D}((u(t), \gamma(t)),(v, \tau))
$$

for every pair $(v, \tau) \in H^{1}(\Omega \backslash \Gamma) \times L^{\infty}(\Gamma)$ such that $v=w(t)$ on $\partial_{D} \Omega$ and $\tau \geq \gamma(t)$;

(b) irreversibility: $t \mapsto \gamma(t)$ is nondecreasing;

(c) energy balance: the function $t \mapsto \partial_{t} \tilde{E}(t,(u(t), \gamma(t)))$ belongs to $L^{1}([0, T])$ and

$$
\tilde{E}(t,(u(t), \gamma(t)))+\operatorname{Diss}_{\mathcal{D}}((u, \gamma) ;[0, t])=\tilde{E}(0,(u(0), \gamma(0)))+\int_{0}^{t} \partial_{s} \tilde{E}(s,(u(s), \gamma(s))) \mathrm{d} s
$$

for every $t \in[0, T]$,

where the dissipation $\operatorname{Diss}_{\mathcal{D}}((u, \gamma) ;[0, t])$ along the curve $s \mapsto(u(s), \gamma(s))$ between 0 and $t$ is defined as the total variation with respect to the "metric" $\mathcal{D}$, so that $\operatorname{Diss}_{\mathcal{D}}((u, \gamma) ;[0, t])=\int_{\Gamma}(\varphi(0, \gamma(t))-\varphi(0, \gamma(0))) \mathrm{d} \mathcal{H}^{N-1}$.

Following $[4,7]$, in order to prove an existence result for the irreversible quasistatic evolution, we perform a time discretization procedure. We define discrete-time evolutions $\left(u_{k}(t), \gamma_{k}(t)\right)$ by solving incremental minimum problems, and we let the time step go to 0 .

The main difficulty in passing to the continuous-time limit is the lack of compactness of the internal variables $\gamma_{k}$. In the particular case of a constant unloading response (i.e., $\varphi$ does not depend on $[u]$ ) this problem was overcome in [5] by defining a suitable notion of convergence, inspired by the $\sigma$-convergence introduced in [6]. We choose here a different approach based on the use of Young measures. We are thus lead to consider in Section 3 a weaker formulation of the problem in which the internal variable is a Young measure $\nu$. The total energy associated to an admissible configuration $(u, \nu)$ becomes

$$
E(u, \nu):=\frac{1}{2} \int_{\Omega \backslash \Gamma}|\nabla u|^{2} \mathrm{~d} x+\int_{\Gamma} \int_{\mathbb{R}} \varphi(|[u]|, \xi) \mathrm{d} \nu_{x}(\xi) \mathrm{d} \mathcal{H}^{N-1} .
$$

To deal with irreversibility and unilateral global stability, we introduce an order relation " measures (see Def. 3.10) and prove an extension of Helly's selection principle to this framework (see Thm. 3.20).

Thus, a Young measure solution to the irreversible quasistatic evolution problem is a function $t \mapsto\left(u(t), \nu^{t}\right)$ such that for every $t \in[0, T]$ the internal variable $\nu^{t}$ is a Young measure and

$\left(\mathrm{a}^{\prime}\right)$ (unilateral) global stability: for every $t \in[0, T]$

$$
E\left(u(t), \nu^{t}\right) \leq E(v, \mu)
$$

for every pair $(v, \mu)$ with $v \in H^{1}(\Omega \backslash \Gamma), v=w(t)$ on $\partial_{D} \Omega$ and $\nu^{t} \preceq \mu$;

$\left(\mathrm{b}^{\prime}\right)$ irreversibility: $t \mapsto \nu^{t}$ is nondecreasing with respect to $\preceq$;

( $\left.{ }^{\prime}\right)$ energy balance: the function $t \mapsto \int_{\Omega \backslash \Gamma} \nabla u(t) \cdot \nabla \dot{w}(t) \mathrm{d} x$ belongs to $L^{1}([0, T])$ and

$$
E\left(u(t), \nu^{t}\right)=E\left(u(0), \nu^{0}\right)+\int_{0}^{t} \int_{\Omega \backslash \Gamma} \nabla u(s) \cdot \nabla \dot{w}(s) \mathrm{d} x \mathrm{~d} s
$$

for every $t \in[0, T]$.

The theoretical tools we develop allow us to prove in Theorem 3.30 the existence of such a solution. In particular, to pass to the limit from the discrete-time problems to the continuous-time evolution we use a compactness result which gives the convergence in the sense of Young measures (Thm. 3.5).

In the case of a constant unloading response we prove (see Thm. 5.1) that the solution given by Theorem 3.30 is a concentrated Young measure, thus recovering the result in [5]. As a consequence, we are able to show 
that the internal variables $\gamma_{k}$ actually converge in measure to the continuous-time internal variable $\gamma$ (see Rem. 5.2), which improves the $\sigma$-convergence obtained in [5].

For a general unloading response, it is still an open question whether or not $\left(\mathrm{a}^{\prime}\right)-\left(\mathrm{c}^{\prime}\right)$ admits a concentrated Young measure solution. Nevertheless, we show that the a priori bounds available for the discrete-time evolutions are not enough to guarantee that the limit measure is concentrated (Prop. 6.1). Hence, to give a full answer to this question one should probably exploit the minimality properties of the discrete-time evolutions.

At the end of the paper we give the Euler-Lagrange conditions for the unilateral global stability in two equivalent formulations (Props. 7.1 and 7.2).

The paper is organized as follows. In Section 2 we fix the notations and the details of the problem. Section 3 contains the results needed to give a formulation in the setting of Young measures. The existence result for irreversible quasistatic evolutions in the sense of Young measures is proved in Section 4. The case of constant unloading response is the subject of Section 5. In Section 6 we show with an example that the a priori estimates on the discrete-time variables are not sufficient to guarantee that the limit measure is localized. Finally, Section 7 is devoted to the necessary conditions for the unilateral global stability.

\section{Setting of the Problem}

In this section we give some basic definitions and we introduce the problem. We will use the following notations:

$-\mathcal{T}:=\left\{(y, z) \in \mathbb{R}^{2}: y>0, z>0, z>y\right\}$

- $\mathcal{L}^{k}$ is the Lebesgue measure in $\mathbb{R}^{k}, k \in \mathbb{N}$;

- $\mathcal{H}^{N-1}$ is the $(N-1)$-dimensional Hausdorff measure in $\mathbb{R}^{N}$.

For every set $A \subset \mathbb{R}^{N}$ :

$-1_{A}$ is the characteristic function of $A$;

- $A^{c}$ is the complement of $A$ in $\mathbb{R}^{N}$;

- $\mathcal{D}^{\prime}(A)$ is the space of distributions on $A$;

- $\mathcal{B}(A)$ is the $\sigma$-algebra of Borel sets in $A$;

- $\mathcal{M}_{b}^{+}(A)$ is the set of the nonnegative bounded Radon measures on $A$.

Let $\Omega$ be a bounded open set in $\mathbb{R}^{N}, N \geq 2$, with Lipschitz boundary, and let $\Gamma \subset \mathbb{R}^{N}$ be a Lipschitz closed set such that $0<\mathcal{H}^{N-1}(\Gamma \cap \bar{\Omega})<+\infty$ and $\Omega \backslash \Gamma=\Omega^{+} \cup \Omega^{-}$, with $\Omega^{ \pm}$disjoint open connected sets with Lipschitz boundary. We will prescribe time dependent boundary displacements on $\partial_{D} \Omega \subset \partial \Omega$, where

$$
\partial_{D} \Omega=\Lambda_{D}^{+} \cup \Lambda_{D}^{-}
$$

with $\Lambda_{D}^{+}$and $\Lambda_{D}^{-}$non empty relatively open, connected, Lipschitz sets. We also assume that $\Lambda_{D}^{ \pm} \subset \subset\left(\partial \Omega^{ \pm} \backslash \Gamma\right)$, from which it follows that $\partial_{D} \Omega$ is well-separated from $\Gamma$. With $\mathbf{n}$ we denote the inner unit normal vector to $\partial \Omega$, defined $\mathcal{H}^{N-1}$-a.e. in $\partial \Omega$. We will also write $\mathbf{n}$ for the inner unit normal vector to $\partial \Omega^{+}$.

Let us fix a time interval $[0, T]$, with $T>0$, and let $w \in H^{1}\left((0, T) ; H^{1}(\Omega)\right)$ be the boundary displacement. Thus, the time derivative $\dot{w}$ of $w$ belongs to the space $L^{2}\left((0, T) ; H^{1}(\Omega)\right)$. We will assume that $\sup _{t \in[0, T]}\|w(t)\|_{L^{\infty}(\Omega)}=: M<+\infty$.

Let $B \subset \mathbb{R}^{N}$ be an open bounded set and let $S \subset \partial B$ be relatively open and Lipschitz. We set

$$
H_{0}^{1}(B, S):=\left\{\psi \in H^{1}(B): \psi=0 \text { on } S\right\}
$$

The symbol $\|\cdot\|$ stands for the standard norm in $L^{2}(\Omega)$ or $L^{2}\left(\Omega \backslash \Gamma ; \mathbb{R}^{N}\right)$, depending on the context. Moreover, the brackets $\langle\cdot, \cdot\rangle$ denote the dual pairing between $H^{-\frac{1}{2}}(\Gamma)$ and $H^{\frac{1}{2}}(\Gamma)$. For every function $v \in H^{1}(\Omega \backslash \Gamma)$, we will use the notation $[v]:=v^{+}-v^{-}$, where $v^{ \pm}$is the trace on $\Gamma$ of the restriction of $v$ to $\Omega^{ \pm}$. Let

$$
L^{\infty}(\Gamma)^{+}:=\left\{\tau \in L^{\infty}(\Gamma): \tau \geq 0 \quad \mathcal{H}^{N-1} \text {-a.e. on } \Gamma\right\} \text {. }
$$


For $t \in[0, T]$, the class $\mathcal{A}(t, w)$ of admissible displacements at time $t$ is defined as

$$
\mathcal{A}(t, w):=\left\{(v, \tau) \in H^{1}(\Omega \backslash \Gamma) \times L^{\infty}(\Gamma)^{+}: v=w(t) \text { on } \partial_{D} \Omega,|[v]| \leq \tau \quad \mathcal{H}^{N-1} \text {-a.e. on } \Gamma\right\}
$$

Recalling the considerations made in the introduction, the total energy associated at time $t$ to a pair $(v, \tau) \in$ $\mathcal{A}(t, w)$ is

$$
E(v, \tau):=\frac{1}{2} \int_{\Omega \backslash \Gamma}|\nabla v|^{2} \mathrm{~d} x+\int_{\Gamma} \varphi(|[v]|, \tau) \mathrm{d} \mathcal{H}^{N-1} .
$$

We will assume that $\varphi \in C^{1}(\overline{\mathcal{T}} \backslash\{0\}) \cap C^{0}(\overline{\mathcal{T}})$ and:

$-\varphi(z, z)=g(z)$ for every $z \in[0,+\infty)$,

$-\varphi(\cdot, z)$ is nondecreasing and convex for every $z \in(0,+\infty)$,

$-\varphi(y, \cdot)$ is nondecreasing for every $y \in[0,+\infty)$,

where $g:[0,+\infty) \rightarrow[0,+\infty)$ is a $C^{1}$, nondecreasing, bounded, concave function with $g(0)=0$. We will denote by $\sigma:=g^{\prime}\left(0^{+}\right) \in(0,+\infty)$ the slope of the function $g$ at 0 .

We can give now the definition of (unilateral) global stability.

Definition 2.1. A pair $(u, \gamma)$ is globally stable at time $t \in[0, T]$ if

$-(u, \gamma) \in \mathcal{A}(t, w)$

- $E(u, \gamma) \leq E(v, \tau)$ for every $(v, \tau) \in \mathcal{A}(t, w)$ with $\tau \geq \gamma$.

Remark 2.2. One can see (see also Rem. 3.22) that the two conditions of Definition 2.1 are equivalent to the following:

$-(u, \gamma) \in \mathcal{A}(t, w)$

- $E(u, \gamma) \leq E(v, \gamma \vee|[v]|)$ for every $v \in H^{1}(\Omega \backslash \Gamma)$ such that $v=w(t)$ on $\partial_{D} \Omega$.

Thanks to Remark 2.2, for every fixed $t \in[0, T]$ the existence of a globally stable pair $(u, \gamma)$ at time $t$ follows by the direct method of the Calculus of Variations.

Remark 2.3. Suppose that a pair $(u(t), \gamma(t))$ is globally stable at time $t$ for every $t \in[0, T]$. Choosing $(w(t), \gamma(t))$ as test pair, we have that there exists a constant $C>0$ such that

$$
\frac{1}{2} \int_{\Omega \backslash \Gamma}|\nabla u(t)|^{2} \mathrm{~d} x+\int_{\Gamma} \varphi(|[u(t)]|, \gamma(t)) \mathrm{d} \mathcal{H}^{N-1} \leq \frac{1}{2} \int_{\Omega \backslash \Gamma}|\nabla w(t)|^{2} \mathrm{~d} x+\int_{\Gamma} \varphi(0, \gamma(t)) \mathrm{d} \mathcal{H}^{N-1} \leq C,
$$

since $w \in H^{1}\left((0, T) ; H^{1}(\Omega)\right)$ and $\varphi$ is bounded. In particular, $\|\nabla u(t)\| \leq C$ for $t \in[0, T]$ and, by Poincaré inequality,

$$
\|u(t)\|_{H^{1}(\Omega \backslash \Gamma)} \leq C,
$$

where $C$ denotes different constants independent of $t \in[0, T]$. By a truncation argument, from the fact that $\sup _{t \in[0, T]}\|w(t)\|_{L^{\infty}(\Omega)} \leq M$ it follows that $\|u(t)\|_{L^{\infty}(\Omega)} \leq M$ and, in turn, $\|[u(t)]\|_{L^{\infty}(\Gamma)} \leq 2 M$ for every $t \in[0, T]$. Since for every $y \in[0,+\infty)$ the function $\varphi(y, \cdot)$ is nondecreasing, defining $\tilde{\gamma}(t):=\gamma(t) \wedge 2 M$ one has

$$
E(u(t), \tilde{\gamma}(t)) \leq E(u(t), \gamma(t))
$$

so that the pair $(u(t), \tilde{\gamma}(t))$ is still globally stable at time $t$.

In the sequel, thanks to the previous remark, we will always assume $\gamma \in[0,2 M]$. Finally, we give the definition of irreversible quasistatic evolution.

Definition 2.4. An irreversible quasistatic evolution is a function $t \mapsto(u(t), \gamma(t))$ from $[0, T]$ to $H^{1}(\Omega \backslash \Gamma) \times$ $L^{\infty}(\Gamma)^{+}$such that the following conditions are satisfied:

(a) (unilateral) global stability: $(u(t), \gamma(t))$ is globally stable at time $t$ for every $t \in[0, T]$; 
(b) irreversibility: $t \mapsto \gamma(t)$ is nondecreasing;

(c) energy balance: the function $t \mapsto \int_{\Omega \backslash \Gamma} \nabla u(t) \cdot \nabla \dot{w}(t) \mathrm{d} x$ belongs to $L^{1}([0, T])$ and for every $t \in[0, T]$

$$
E(u(t), \gamma(t))=E(u(0), \gamma(0))+\int_{0}^{t} \int_{\Omega \backslash \Gamma} \nabla u(s) \cdot \nabla \dot{w}(s) \mathrm{d} x \mathrm{~d} s .
$$

Unfortunately, we are not able to provide an existence result for such evolutions in the general case. This will be proved only when the unloading response is constant (see Sect. 5). For this reason, we extend the definition of irreversible quasistatic evolution to the setting of Young measures.

\section{Irreversible QuAsistatic EVOlution in the SetTing of Young measures}

We give here a weak formulation of irreversible quasistatic evolution, in the framework of Young measures. In the whole section we will assume that $c$ and $d$ are fixed real numbers such that $-\infty<c<d<+\infty$.

\subsection{Young measures}

We recall now some definitions and properties of Young measures that will be useful in the sequel. For the results contained in this subsection, see [10]. We set:

$$
\mathcal{P}([c, d]):=\{\text { probability measures on }[c, d]\}=\left\{\nu \in \mathcal{M}_{b}^{+}([c, d]): \nu([c, d])=1\right\} .
$$

We recall the following useful characterization of probability measures in $[c, d]$ (see [3], Prop. 1.3.8, where a slightly different version of the proposition is stated).

Proposition 3.1. For each nonincreasing, left continuous function $F:[c, d] \rightarrow[0,1]$ that satisfies $F(c)=1$, there is a unique measure $\nu \in \mathcal{P}([c, d])$ such that the equality $F(a)=\nu([a, d])$ holds at each $a \in[c, d]$.

We now give the definition of measurable family in $\mathcal{P}([c, d])$.

Definition 3.2. We say that a family $\left(\nu_{x}\right)_{x \in \Gamma}$ in $\mathcal{P}([c, d])$ is measurable if for every $A \in \mathcal{B}([c, d])$ the scalar function $x \mapsto \nu_{x}(A)$ is measurable with respect to the $\sigma$-algebra $\mathcal{B}(\Gamma)$. The set of all such families is denoted with $\mathcal{P}(\Gamma,[c, d])$.

Definition 3.3. We define the collection of Young measures on $\Gamma \times[c, d]$ with respect to the measure $\mathcal{H}^{N-1}$ as the set

$$
\mathcal{Y}\left(\Gamma, \mathcal{H}^{N-1},[c, d]\right):=\left\{\lambda \in \mathcal{M}_{b}^{+}(\Gamma \times[c, d]): \quad \lambda(A \times[c, d])=\mathcal{H}^{N-1}(A) \quad \forall A \in \mathcal{B}(\Gamma)\right\} .
$$

We introduce now a topology in $\mathcal{Y}\left(\Gamma, \mathcal{H}^{N-1},[c, d]\right)$.

Definition 3.4. Let $\left(\lambda_{j}\right)$ be a sequence in $\mathcal{Y}\left(\Gamma, \mathcal{H}^{N-1},[c, d]\right)$. We say that $\left(\lambda_{j}\right) w^{*}$-converges to $\lambda \in \mathcal{Y}\left(\Gamma, \mathcal{H}^{N-1}\right.$, $[c, d])$ if

$$
\int_{\Gamma \times[c, d]} f \mathrm{~d} \lambda_{j} \rightarrow \int_{\Gamma \times[c, d]} f \mathrm{~d} \lambda
$$

for every $f \in C^{0}(\Gamma \times[c, d])$. We will also write $\lambda_{j} \stackrel{\mathcal{Y}}{\longrightarrow} \lambda$.

The following compactness result holds.

Theorem 3.5 (compactness theorem). $\mathcal{Y}\left(\Gamma, \mathcal{H}^{N-1},[c, d]\right)$ endowed with the $w^{*}$-topology is sequentially compact.

Next theorem gives the connection between Young measures and measurable families in $\mathcal{P}([c, d])$. 
Theorem 3.6 (disintegration theorem). The map $\Phi: \mathcal{P}(\Gamma,[c, d]) \rightarrow \mathcal{Y}\left(\Gamma, \mathcal{H}^{N-1},[c, d]\right)$ which associates to $\left(\nu_{x}\right)_{x \in \Gamma}$ the Young measure $\lambda$ given by

$$
\lambda(G):=\int_{\Gamma} \int_{[c, d]} 1_{G}(x, \xi) \mathrm{d} \nu_{x}(\xi) \mathrm{d} \mathcal{H}^{N-1}(x) \quad G \in \mathcal{B}(\Gamma \times[c, d])
$$

induces a bijection between $\mathcal{P}(\Gamma,[c, d]) / \sim$ and $\mathcal{Y}\left(\Gamma, \mathcal{H}^{N-1},[c, d]\right)$, where $\sim$ is the equivalence relation defined by

$$
\nu_{1} \sim \nu_{2} \Longleftrightarrow\left(\nu_{1}\right)_{x}=\left(\nu_{2}\right)_{x} \text { for } \mathcal{H}^{N-1} \text {-a.e. } x \in \Gamma \text {. }
$$

Definition 3.7. Let $u: \Gamma \rightarrow[c, d]$ be a measurable function. We denote by $\delta_{u}$ the element of $\mathcal{P}(\Gamma,[c, d])$ defined by

$$
\left(\delta_{u}\right)_{x}=\delta_{u(x)} \quad \text { for } \mathcal{H}^{N-1} \text {-a.e. } x \in \Gamma .
$$

Identifying $\mathcal{P}(\Gamma,[c, d]) / \sim$ and $\mathcal{Y}\left(\Gamma, \mathcal{H}^{N-1},[c, d]\right)$ in the sense of Theorem 3.6, we will refer to $\delta_{u}$ as the concentrated Young measure associated to $u$.

The following theorem (see [10], Thm. 17) will be useful in the proof of the main result of the paper.

Theorem 3.8. Let $\left(u_{k}\right)_{k \in \mathbb{N}}$ be a sequence of measurable functions from $\Gamma$ to $[c, d]$. Suppose that $\delta_{u_{k}} \stackrel{\mathcal{Y}}{\longrightarrow} \nu$ for some $\nu \in \mathcal{Y}\left(\Gamma, \mathcal{H}^{N-1},[c, d]\right)$. Let $\phi: \Gamma \times[c, d] \rightarrow \mathbb{R}$ be a Carathéodory function, that is, $\phi$ is measurable and $\phi(x, \cdot)$ is continuous for $\mathcal{H}^{N-1}$-a.e. $x \in \Gamma$. In addition, assume that $x \mapsto \phi\left(x, u_{k}(x)\right)$ is uniformly integrable. Then $\phi(x, \cdot)$ is $\nu_{x}$-integrable for $\mathcal{H}^{N-1}$-a.e. $x \in \Gamma$ and

$$
\lim _{k \rightarrow+\infty} \int_{\Gamma} \phi\left(x, u_{k}(x)\right) \mathrm{d} \mathcal{H}^{N-1}(x)=\int_{\Gamma} \int_{[c, d]} \phi(x, \xi) \mathrm{d} \nu_{x}(\xi) \mathrm{d} \mathcal{H}^{N-1}(x) .
$$

We conclude the subsection with a proposition that will be used in Remark 5.2.

Proposition 3.9. Let $u, u_{k}: \Gamma \rightarrow[c, d]$ be measurable functions. Then

$$
u_{k} \rightarrow u \text { in measure } \Longleftrightarrow \delta_{u_{k}} \stackrel{\mathcal{Y}}{\longrightarrow} \delta_{u} .
$$

\subsection{Partial ordering for Young measures}

Let us consider the following order relation in $\mathcal{Y}\left(\Gamma, \mathcal{H}^{N-1},[c, d]\right)$.

Definition 3.10. Let $\nu_{1}, \nu_{2} \in \mathcal{Y}\left(\Gamma, \mathcal{H}^{N-1},[c, d]\right)$. We say that $\nu_{1} \preceq \nu_{2}$ if for every $A \in \mathcal{B}(\Gamma)$ and for every $a \in[c, d]$ there holds

$$
\int_{A}\left(\nu_{1}\right)_{x}([a, d]) \mathrm{d} \mathcal{H}^{N-1}(x) \leq \int_{A}\left(\nu_{2}\right)_{x}([a, d]) \mathrm{d} \mathcal{H}^{N-1}(x) .
$$

Proposition 3.11. Let $\nu_{1}, \nu_{2} \in \mathcal{Y}\left(\Gamma, \mathcal{H}^{N-1},[c, d]\right)$. The following conditions are equivalent:

(i) $\nu_{1} \preceq \nu_{2}$;

(ii) $\left(\nu_{1}\right)_{x}([a, d]) \leq\left(\nu_{2}\right)_{x}([a, d])$ for every $a \in[c, d]$, for $\mathcal{H}^{N-1}$-a.e. $x \in \Gamma$;

(iii) for every $f \in C^{0}(\Gamma \times[c, d])$ nondecreasing with respect to the second variable

$$
\int_{\Gamma} \int_{[c, d]} f(x, \xi) \mathrm{d}\left(\nu_{1}\right)_{x} \mathrm{~d} \mathcal{H}^{N-1}(x) \leq \int_{\Gamma} \int_{[c, d]} f(x, \xi) \mathrm{d}\left(\nu_{2}\right)_{x} \mathrm{~d} \mathcal{H}^{N-1}(x) .
$$

The proof follows from standard approximation and localization techniques.

Remark 3.12. In particular, if $\nu_{1}=\delta_{f_{1}}$ and $\nu_{2}=\delta_{f_{2}}$, with $f_{1}, f_{2}: \Gamma \rightarrow[c, d]$ measurable functions, then $\nu_{1} \preceq \nu_{2}$ is equivalent to

$$
f_{1} \leq f_{2} \quad \mathcal{H}^{N-1} \text {-a.e. on } \Gamma \text {. }
$$


Remark 3.13. For every $\nu \in \mathcal{P}(\Gamma,[c, d])$ and $a \in[c, d]$ let $z_{a}^{\nu}$ be the measurable function $z_{a}^{\nu}: \Gamma \rightarrow[0,1]$ defined by $z_{a}^{\nu}(x):=\nu_{x}([a, d])$ for $\mathcal{H}^{N-1}$-a.e. $x \in \Gamma$. Using this notation, $\nu_{1} \preceq \nu_{2}$ is equivalent to

$$
z_{a}^{\nu_{1}} \leq z_{a}^{\nu_{2}} \quad \mathcal{H}^{N-1} \text {-a.e. on } \Gamma \text {, for every } a \in[c, d] .
$$

Remark 3.14. From condition (iii) of Proposition 3.11 it follows that the inequality between Young measures is preserved under the limit operation. More precisely, let $\left(\nu_{n}\right)$ and $\left(\mu_{n}\right)$ be two sequences of Young measures such that $\nu_{n} \stackrel{\mathcal{Y}}{\longrightarrow} \nu^{*}, \mu_{n} \stackrel{\mathcal{Y}}{\longrightarrow} \mu^{*}$ and $\nu_{n} \preceq \mu_{n}$ for every $n \in \mathbb{N}$. Then $\nu^{*} \preceq \mu^{*}$.

Definition 3.15. Let $D \subset[0, T]$ and let $\nu: D \rightarrow \mathcal{Y}\left(\Gamma, \mathcal{H}^{N-1},[c, d]\right)$ be a function that associates to every $s \in D$ a Young measure $\nu^{s}$. We say that $\bar{\nu} \in \mathcal{Y}\left(\Gamma, \mathcal{H}^{N-1},[c, d]\right)$ is the supremum over $s \in D$ of the family of measures $\left\{\nu^{s}: s \in D\right\}$ if the following two conditions hold:

(i) $\nu^{s} \preceq \bar{\nu}$ for every $s \in D$;

(ii) if $\mu \in \mathcal{Y}\left(\Gamma, \mathcal{H}^{N-1},[c, d]\right)$ and $\nu^{s} \preceq \mu$ for every $s \in D$, then $\bar{\nu} \preceq \mu$.

We will also write

$$
\bar{\nu}=\sup _{s \in D} \nu^{s}
$$

In the same way one can define the infimum of a family of Young measures.

Proposition 3.16. Let $F=\left\{\nu^{s}: s \in D\right\} \subset \mathcal{Y}\left(\Gamma, \mathcal{H}^{N-1},[c, d]\right)$ be a family of Young measures on $\Gamma \times[c, d]$. Then, the supremum (infimum) of $F$ exists and is unique.

Proof. For every $a \in[c, d]$, we set

$$
f(a, \cdot):=\underset{s \in D}{\operatorname{esssup}} z_{a}^{\nu^{s}}
$$

where the functions $z_{a}^{\nu^{s}}$ are defined in Remark 3.13. We recall that the essential supremum (see [9], Prop. VI-1-1)

$$
z=\underset{i \in I}{\operatorname{ess} \sup } z_{i}
$$

of an arbitrary family $\left(z_{i}\right)_{i \in I}$ in $L^{\infty}(\Gamma)^{+}$is defined as the unique (up to $\mathcal{H}^{N-1}$-equivalence) function in $L^{\infty}(\Gamma)^{+}$ such that

- $z \geq z_{i} \mathcal{H}^{N-1}$-a.e. on $\Gamma$ for every $i \in I$;

- if $q \in L^{\infty}(\Gamma)^{+}$and $q \geq z_{i} \mathcal{H}^{N-1}$-a.e. on $\Gamma$ for every $i \in I$, then $q \geq z \mathcal{H}^{N-1}$-a.e. on $\Gamma$.

By definition of essential supremum, it follows that $f(c, \cdot)=1 \mathcal{H}^{N-1}$-a.e. on $\Gamma$. Moreover, for every $a, b \in$ $\{c\} \cup((c, d] \cap \mathbb{Q})$ with $a \leq b$ there exists a set $J^{a, b} \subset \Gamma$ with $\mathcal{H}^{N-1}\left(J^{a, b}\right)=0$ such that

$$
f(a, x) \geq f(b, x) \quad \text { for every } x \in \Gamma \backslash J^{a, b} .
$$

Let us define the set $J \subset \Gamma$ as

$$
J:=\bigcup_{a, b} J^{a, b},
$$

where the union is taken among all $a, b \in\{c\} \cup((c, d] \cap \mathbb{Q})$ with $a \leq b$. Then, $\mathcal{H}^{N-1}(J)=0$ and for every $a, b \in\{c\} \cup((c, d] \cap \mathbb{Q})$ with $a \leq b$ there holds

$$
f(a, x) \geq f(b, x) \quad \text { for every } x \in \Gamma \backslash J .
$$

Let $x \in \Gamma \backslash J$ be chosen arbitrarily. Since $f(\cdot, x)$ is nonincreasing in $\{c\} \cup((c, d] \cap \mathbb{Q})$ we can modify it in such a way that it is left-continuous. We define $\hat{f}(c, x)=1$ and for every $a \in(c, d]$ we set

$$
\hat{f}(a, x):=\lim _{\substack{b \rightarrow a^{-} \\ b \in(c, d] \cap \mathbb{Q}}} f(b, x) .
$$


By construction, the function $x \mapsto \hat{f}(a, x)$ is measurable for every $a \in[c, d]$. Moreover, for $\mathcal{H}^{N-1}$-a.e. $x \in \Gamma$ the function $a \mapsto \hat{f}(a, x)$ is nonincreasing and left-continuous, and $\hat{f}(c, x)=1$. Thus, by Proposition 3.1 for $\mathcal{H}^{N-1}$-a.e. $x \in \Gamma$ there exists a probability measure $\bar{\nu}_{x} \in \mathcal{P}([c, d])$ such that $\hat{f}(a, x)=\bar{\nu}_{x}([a, d])$ for every $a \in[c, d]$. Let us set

$$
\mathcal{G}:=\left\{A \in \mathcal{B}([c, d]): x \mapsto \bar{\nu}_{x}(A) \text { is measurable }\right\}
$$

We want to show that $\mathcal{G}=\mathcal{B}([c, d])$. First of all, observe that by the continuity properties of measures along monotone sequences of sets it follows that $\mathcal{G}$ is a monotone class. Moreover, one can easily check that $\mathcal{G}$ contains the algebra generated by all the intervals $[a, d]$ with $a \in[c, d]$. From the Monotone Class theorem (see e.g. [3]) we get $\mathcal{G}=\mathcal{B}([c, d])$. At this point, by Theorem 3.6 we conclude that $\bar{\nu} \in \mathcal{Y}\left(\Gamma, \mathcal{H}^{N-1},[c, d]\right)$. Using the properties of the essential supremum one can check that, by construction, $\bar{\nu}$ satisfies properties $(i)$ and $(i i)$. Uniqueness follows immediately by property $(i i)$.

Remark 3.17. For every $s \in D$, let $f^{s}: \Gamma \rightarrow[c, d]$ be a measurable function and set $\nu^{s}=\delta_{f}$. Then

$$
\sup _{s \in D} \nu^{s}=\sup _{s \in D} \delta_{f s}=\delta_{\operatorname{ess} \sup _{s \in D} f^{s}}
$$

Remark 3.18. When dealing with a finite number $m \in \mathbb{N}$ of Young measures, we will simply use the notation $\sup _{i=1, \ldots, m} \nu_{i}=: \nu_{1} \vee \ldots \vee \nu_{m}$. In the case $m=2$, when $\nu_{1}=\delta_{f}$ with $f: \Gamma \rightarrow[c, d]$ a measurable function, there holds

$$
\left(\delta_{f} \vee \nu_{2}\right)_{x}=\nu_{2}([c, f(x)]) \delta_{f(x)}+\left.\left(\nu_{2}\right)_{x}\right|_{(f(x), d]} \quad \text { for } \mathcal{H}^{N-1} \text {-a.e. } x \in \Gamma,
$$

where $\left.\left(\nu_{2}\right)_{x}\right|_{(f(x), d]}$ denotes the restriction of the measure $\left(\nu_{2}\right)_{x}$ to the interval $(f(x), d]$. The previous inequality can be easily proved evaluating the two measures in the sets $[a, d]$ for every $a \in[c, d]$.

We state now a preliminary lemma and a useful version of the Helly's selection principle. For similar results in the context of nondecreasing set functions, see [4], Lemma 6.2 and Theorem 6.3.

Lemma 3.19. Let $\nu_{1}, \nu_{2}:[0, T] \rightarrow \mathcal{Y}\left(\Gamma, \mathcal{H}^{N-1},[c, d]\right)$ be two nondecreasing functions such that

$$
\nu_{1}^{s} \preceq \nu_{2}^{t} \quad \text { and } \quad \nu_{2}^{s} \preceq \nu_{1}^{t}
$$

for every $s, t \in[0, T]$ with $s<t$. Let $D$ be the set of points $t \in[0, T]$ such that $\nu_{1}^{t}=\nu_{2}^{t}$. Then, $[0, T] \backslash D$ is at most countable.

Proof. Let us fix $A \subset \Gamma$ relatively open. For $i=1,2$, consider the functions $f_{i}:[c, d] \times[0, T] \rightarrow \mathbb{R}$ defined by

$$
f_{i}(a, t):=\int_{A}\left(\nu_{i}^{t}\right)_{x}([a, d]) \mathrm{d} \mathcal{H}^{N-1}
$$

By the continuity properties of measures along decreasing sequences of sets, it follows that $f_{i}(\cdot, t)(i=1,2)$ is left-continuous for every $t \in[0, T]$. Moreover, $f_{i}(a, \cdot)(i=1,2)$ is nondecreasing for every $a \in[c, d]$. Then, for every $a \in\{c\} \cup((c, d] \cap \mathbb{Q})$ there exists an at most countable set $J_{a}^{A} \subset[0, T]$ such that both $f_{1}(a, \cdot)$ and $f_{2}(a, \cdot)$ are continuous at every point of $[0, T] \backslash J_{a}^{A}$. Let $J^{A}$ be the (at most countable) set defined by $J^{A}:=\bigcup_{a} J_{a}^{A}$, where the union is taken among all $a \in\{c\} \cup((c, d] \cap \mathbb{Q})$. Then, using (3.1) we get that for every $t \in[0, T] \backslash J^{A}$ there holds $f_{1}(a, t)=f_{2}(a, t)$ for every $a \in\{c\} \cup((c, d] \cap \mathbb{Q})$ and, by the left-continuity, for every $a \in[c, d]$. That is, for every $t \in[0, T] \backslash J^{A}$ and for every $a \in[c, d]$

$$
\int_{A}\left(\nu_{1}^{t}\right)_{x}([a, d]) \mathrm{d} \mathcal{H}^{N-1}=\int_{A}\left(\nu_{2}^{t}\right)_{x}([a, d]) \mathrm{d} \mathcal{H}^{N-1}
$$

Let us consider a countable $\pi$-system $\left(A_{n}\right)_{n \in \mathbb{N}}$ on $\Gamma$ that generates the $\sigma$-algebra $\mathcal{B}(\Gamma)$. We recall that a $\pi$-system on $\Gamma$ is a family of subsets of $\Gamma$ which is closed under the formation of finite intersections and contains $\Gamma$. Analogously to what we have done when $A \in \mathcal{B}(\Gamma)$ was fixed, for every $n \in \mathbb{N}$ we can define an at most 
countable set $J^{A_{n}} \subset[0, T]$ such that relation (3.2) holds with $A$ replaced by $A_{n}$ for every $t \in[0, T] \backslash J^{A_{n}}$. Defining $J:=\bigcup_{n \in \mathbb{N}} J^{A_{n}}$ we get that for every $t \in[0, T] \backslash J$ there holds

$$
\int_{A_{n}}\left(\nu_{1}^{t}\right)_{x}([a, d]) \mathrm{d} \mathcal{H}^{N-1}=\int_{A_{n}}\left(\nu_{2}^{t}\right)_{x}([a, d]) \mathrm{d} \mathcal{H}^{N-1}
$$

for every $a \in[c, d]$ and $n \in \mathbb{N}$. By [3], Corollary 1.6.2, we obtain that $\nu_{1}^{t}=\nu_{2}^{t}$ for every $t \in[0, T] \backslash J$, hence the thesis with $D=[0, T] \backslash J$.

Theorem 3.20 (Helly's selection principle for Young measures). Let $\left(\nu_{n}\right)$ be a sequence of nondecreasing functions from $[0, T]$ into $\mathcal{Y}\left(\Gamma, \mathcal{H}^{N-1},[c, d]\right)$. Then there exists a subsequence, still denoted by $\left(\nu_{n}\right)$, and a nondecreasing function $\nu:[0, T] \rightarrow \mathcal{Y}\left(\Gamma, \mathcal{H}^{N-1},[c, d]\right)$, such that $\nu_{n}^{t} \stackrel{\mathcal{Y}}{\longrightarrow} \nu^{t}$ for every $t \in[0, T]$.

Proof. Let $D_{1}$ be a countable dense set of $(0, T)$. Using the compactness Theorem 3.5, Remark 3.14 and a diagonal argument, we find a subsequence, still denoted by $\left(\nu_{n}\right)$, and a nondecreasing function $\nu: D_{1} \rightarrow$ $\mathcal{Y}\left(\Gamma, \mathcal{H}^{N-1},[c, d]\right)$ such that $\nu_{n}^{t} \stackrel{\mathcal{Y}}{\longrightarrow} \nu^{t}$ for every $t \in D_{1}$. Let $\nu_{-}:(0, T] \rightarrow \mathcal{Y}\left(\Gamma, \mathcal{H}^{N-1},[c, d]\right)$ and $\nu_{+}:[0, T) \rightarrow$ $\mathcal{Y}\left(\Gamma, \mathcal{H}^{N-1},[c, d]\right)$ be the nondecreasing functions defined by

$$
\nu_{-}^{t}:=\sup _{\substack{s \in D_{1} \\ s<t}} \nu^{s} \quad \text { for } t \in(0, T] \quad \text { and } \quad \nu_{+}^{t}:=\inf _{\substack{s \in D_{1} \\ s>t}} \nu^{s} \quad \text { for } t \in[0, T) .
$$

Let $D$ be the set of points $t \in[0, T]$ such that $\nu_{-}^{t}=\nu_{+}^{t}$. As $\nu_{-}^{t}$ and $\nu_{+}^{t}$ satisfy (3.1), by Lemma 3.19 the set $[0, T] \backslash D$ is at most countable. Since $\nu_{-}^{t} \preceq \nu^{t} \preceq \nu_{+}^{t}$ for every $t \in D_{1}$, we have $\nu^{t}=\nu_{-}^{t}=\nu_{+}^{t}$ for every $t \in D \cap D_{1}$. For every $t \in D \backslash D_{1}$ we define $\nu^{t}:=\nu_{-}^{t}=\nu_{+}^{t}$. Let us show that this is a good choice. Suppose $t \in D \backslash D_{1}$ is fixed. By the compactness Theorem 3.5 we may assume that $\nu_{n}^{t}$ converges, up to subsequences, to a measure $\nu^{*} \in \mathcal{Y}\left(\Gamma, \mathcal{H}^{N-1},[c, d]\right)$. For every $s_{1}, s_{2} \in D_{1}$ with $s_{1}<t<s_{2}$, by Remark 3.14 we have $\nu^{s_{1}} \preceq \nu^{*} \preceq \nu^{s_{2}}$. Hence, it follows that $\nu_{-}^{t} \preceq \nu^{*} \preceq \nu_{+}^{t}$ and, by the definition of $D, \nu_{-}^{t}=\nu^{*}=\nu^{t}=\nu_{+}^{t}$. Therefore $\nu_{n}^{t} \stackrel{\mathcal{Y}}{\longrightarrow} \nu^{t}$ for every $t \in D \cup D_{1}$. Since $[0, T] \backslash\left(D \cup D_{1}\right)$ is at most countable, by a diagonal argument we find a further subsequence, which we still denote by $\left(\nu_{n}\right)$, and a function $\nu:[0, T] \backslash\left(D \cup D_{1}\right) \rightarrow \mathcal{Y}\left(\Gamma, \mathcal{H}^{N-1},[c, d]\right)$ such that $\nu_{n}^{t} \stackrel{\mathcal{Y}}{\longrightarrow} \nu^{t}$ for every $t \in[0, T] \backslash\left(D \cup D_{1}\right)$. Therefore $\nu_{n}^{t} \stackrel{\mathcal{Y}}{\longrightarrow} \nu^{t}$ for every $t \in[0, T]$, and this implies that $\nu$ is nondecreasing in $[0, T]$.

\subsection{Global stability for Young measures}

In view of Remark 2.3, all the Young measures we will consider belong to $\mathcal{Y}\left(\Gamma, \mathcal{H}^{N-1},[0,2 M]\right)$. For $t \in[0, T]$ the class $a(t, w)$ of admissible configurations at time $t$ is defined as

$$
a(t, w):=\left\{(v, \mu) \in H^{1}(\Omega \backslash \Gamma) \times \mathcal{Y}\left(\Gamma, \mathcal{H}^{N-1},[0,2 M]\right): v=w(t) \text { on } \partial_{D} \Omega, \delta_{|[v]|} \preceq \mu\right\}
$$

The total energy associated at time $t$ to a pair $(v, \mu) \in a(t, w)$ is

$$
E(v, \mu):=\frac{1}{2} \int_{\Omega \backslash \Gamma}|\nabla v|^{2} \mathrm{~d} x+\int_{\Gamma} \int_{[0,2 M]} \varphi(|[v]|, \xi) \mathrm{d} \mu_{x}(\xi) \mathrm{d} \mathcal{H}^{N-1} .
$$

In this setting, we can give the definition of (unilateral) global stability.

Definition 3.21. A pair $(u, \nu)$ is said to be globally stable in the sense of Young measures at time $t \in[0, T]$ if $(u, \nu) \in a(t, w)$ and

$$
E(u, \nu) \leq E(v, \mu)
$$

for every $(v, \mu) \in a(t, w)$ with $\nu \preceq \mu$. 
Remark 3.22. It turns out that the previous condition is equivalent to $(u, \nu) \in a(t, w)$ and

$$
E(u, \nu) \leq E\left(v, \nu \vee \delta_{|[v]|}\right)
$$

for every $v \in H^{1}(\Omega \backslash \Gamma)$ such that $v=w(t)$ on $\partial_{D} \Omega$. Indeed, let us assume that (3.4) holds and let $v \in H^{1}(\Omega \backslash \Gamma)$ be such that $v=w(t)$ on $\partial_{D} \Omega$. Then, we have that $\left(v, \nu \vee \delta_{|[v]|}\right) \in a(t, w)$. Hence, condition (3.4) with the test pair $\left(v, \nu \vee \delta_{|[v]|}\right)$ becomes just (3.5).

Let now (3.5) hold and let $(v, \mu) \in a(t, w)$ with $\nu \preceq \mu$. By definition of the set $a(t, w)$ we have also $\delta_{|[v]|} \preceq \mu$. By (3.5), and since $E$ is increasing with respect to the second variable we get

$$
E(u, \nu) \leq E\left(v, \nu \vee \delta_{|[v]|}\right) \leq E\left(v, \mu \vee \delta_{|[v]|}\right)=E(v, \mu)
$$

We also notice that, thanks to Remark 3.18, condition (3.5) can be written as

$\frac{1}{2} \int_{\Omega \backslash \Gamma}|\nabla u|^{2} \mathrm{~d} x+\int_{\Gamma} \int_{[0,2 M]} \varphi(|[u]|, \xi) \mathrm{d} \nu_{x}(\xi) \mathrm{d} \mathcal{H}^{N-1} \leq \frac{1}{2} \int_{\Omega \backslash \Gamma}|\nabla v|^{2} \mathrm{~d} x+\int_{\Gamma} \int_{[0,2 M]} \varphi(|[v]|,|[v]| \vee \xi) \mathrm{d} \nu_{x}(\xi) \mathrm{d} \mathcal{H}^{N-1}$

for every $v \in H^{1}(\Omega \backslash \Gamma)$ such that $v=w(t)$ on $\partial_{D} \Omega$.

\subsection{Main result}

In this subsection we first give the definition of irreversible quasistatic evolution in the setting of Young measures, and then we state the main result of the paper.

Definition 3.23. An irreversible quasistatic evolution in the sense of Young measures is a function $t \mapsto\left(u(t), \nu^{t}\right)$ from $[0, T]$ to $H^{1}(\Omega \backslash \Gamma) \times \mathcal{Y}\left(\Gamma, \mathcal{H}^{N-1},[0,2 M]\right)$ such that the following three conditions are satisfied:

$\left(\mathrm{a}^{\prime}\right)$ (unilateral) global stability: $\left(u(t), \nu^{t}\right)$ is globally stable in the sense of Young measures at time $t$ for every $t \in[0, T]$;

$\left(\mathrm{b}^{\prime}\right)$ irreversibility: $t \mapsto \nu^{t}$ is nondecreasing with respect to $\preceq$;

$\left(\mathrm{c}^{\prime}\right)$ energy balance: the function $t \mapsto \int_{\Omega \backslash \Gamma} \nabla u(t) \cdot \nabla \dot{w}(t) \mathrm{d} x$ belongs to $L^{1}([0, T])$ and for every $t \in[0, T]$

$$
E\left(u(t), \nu^{t}\right)=E\left(u(0), \nu^{0}\right)+\int_{0}^{t} \int_{\Omega \backslash \Gamma} \nabla u(s) \cdot \nabla \dot{w}(s) \mathrm{d} x \mathrm{~d} s .
$$

Remark 3.24. Condition $\left(c^{\prime}\right)$ is equivalent to the following:

$\left(\mathrm{c}^{\prime \prime}\right)$ the function $t \mapsto E\left(u(t), \nu^{t}\right)$ is absolutely continuous in $[0, T]$ and

$$
\frac{\mathrm{d}}{\mathrm{d} t} E\left(u(t), \nu^{t}\right)=\int_{\Omega \backslash \Gamma} \nabla u(t) \cdot \nabla \dot{w}(t) \mathrm{d} x \quad \text { for a.e. } t \in[0, T] .
$$

Hence, we do not exclude the possibility that the configuration $\left(u(t), \nu^{t}\right)$ varies in such a way that, for example, $t \mapsto\|\nabla u(t)\|$ has some jump points. Anyway, the total energy is always an absolutely continuous function of time. Next theorem shows that one inequality in the energy balance is a direct consequence of conditions $\left(\mathrm{a}^{\prime}\right)$ and $\left(\mathrm{b}^{\prime}\right)$.

Remark 3.25. Let us suppose that for every $t \in[0, T]$ the pair $\left(u(t), \nu^{t}\right)$ is globally stable at time $t$. Then, by repeating the arguments of Remark 2.3 we have that (2.2) still holds.

Theorem 3.26. Let $t \mapsto\left(u(t), \nu^{t}\right)$ be a function from $[0, T]$ into $H^{1}(\Omega \backslash \Gamma) \times \mathcal{Y}\left(\Gamma, \mathcal{H}^{N-1},[0,2 M]\right)$ that satisfies the global stability condition $\left(\mathrm{a}^{\prime}\right)$ and the irreversibility condition $\left(\mathrm{b}^{\prime}\right)$ of Definition 3.23. Assume, in addition, 
that $t \mapsto \int_{\Omega \backslash \Gamma} \nabla u(t) \cdot \nabla \dot{w}(t) \mathrm{d} x$ is measurable. Then

$$
E\left(u(t), \nu^{t}\right) \geq E\left(u_{0}, \nu^{0}\right)+\int_{0}^{t} \int_{\Omega \backslash \Gamma} \nabla u(s) \cdot \nabla \dot{w}(s) \mathrm{d} x \mathrm{~d} s
$$

for every $t \in[0, T]$.

Proof. Note that, thanks to Remark 3.25, $t \mapsto \int_{\Omega \backslash \Gamma} \nabla u(t) \cdot \nabla \dot{w}(t) \mathrm{d} x \in L^{1}([0, T])$. Let now $k \in \mathbb{N}$ be fixed. Consider a subdivision $\left(t_{k}^{i}\right)_{0 \leq i \leq n(k)}$ of the interval $[0, t]$ such that $0=t_{k}^{0} \leq t_{k}^{1} \leq \ldots \leq t_{k}^{n(k)-1} \leq t_{k}^{n(k)}=t$ and

$$
\lim _{k \rightarrow+\infty} \max _{1 \leq i \leq n(k)}\left(t_{k}^{i}-t_{k}^{i-1}\right)=0
$$

Let $1 \leq j<n(k)$ be fixed. Thanks to the minimality of the pair $\left(u\left(t_{k}^{j}\right), \nu^{t_{k}^{j}}\right)$, considering $\left(u\left(t_{k}^{j+1}\right)-w\left(t_{k}^{j+1}\right)+\right.$ $\left.w\left(t_{k}^{j}\right), \nu_{k}^{t_{k}^{j+1}}\right)$ as a test pair we get

$$
\begin{aligned}
\frac{1}{2} \int_{\Omega \backslash \Gamma}\left|\nabla u\left(t_{k}^{j}\right)\right|^{2} \mathrm{~d} x+\int_{\Gamma} \int_{[0,2 M]} & \varphi\left(\left|\left[u\left(t_{k}^{j}\right)\right]\right|, \xi\right) \mathrm{d} \nu_{x}^{t_{k}^{j}}(\xi) \mathrm{d} \mathcal{H}^{N-1}(x) \leq \frac{1}{2} \int_{\Omega \backslash \Gamma}\left|\nabla\left(u\left(t_{k}^{j+1}\right)-w\left(t_{k}^{j+1}\right)+w\left(t_{k}^{j}\right)\right)\right|^{2} \mathrm{~d} x \\
& +\int_{\Gamma} \int_{[0,2 M]} \varphi\left(\left|\left[u\left(t_{k}^{j+1}\right)\right]\right|, \xi\right) \mathrm{d} \nu_{x}^{t_{k}^{j+1}}(\xi) \mathrm{d} \mathcal{H}^{N-1}(x) \\
= & \frac{1}{2} \int_{\Omega \backslash \Gamma}\left|\nabla u\left(t_{k}^{j+1}\right)\right|^{2} \mathrm{~d} x+\int_{\Gamma} \int_{[0,2 M]} \varphi\left(\left|\left[u\left(t_{k}^{j+1}\right)\right]\right|, \xi\right) \mathrm{d} \nu_{x}^{t_{k}^{j+1}}(\xi) \mathrm{d} \mathcal{H}^{N-1}(x) \\
& +\int_{\Omega \backslash \Gamma}\left[\left(-\nabla u\left(t_{k}^{j+1}\right)+\frac{1}{2} \int_{t_{k}^{j}}^{t_{k}^{j+1}} \nabla \dot{w}(s) \mathrm{d} s\right) \cdot \int_{t_{k}^{j}}^{t_{k}^{j+1}} \nabla \dot{w}(s) \mathrm{d} s\right] \mathrm{d} x \\
= & \frac{1}{2} \int_{\Omega \backslash \Gamma}\left|\nabla u\left(t_{k}^{j+1}\right)\right|^{2} \mathrm{~d} x+\int_{\Gamma} \int_{[0,2 M]} \varphi\left(\left|\left[u\left(t_{k}^{j+1}\right)\right]\right|, \xi\right) \mathrm{d} \nu_{x}^{t_{k}^{j+1}}(\xi) \mathrm{d} \mathcal{H}^{N-1}(x) \\
& +\int_{t_{k}^{j}}^{t_{k}^{j+1}} \int_{\Omega \backslash \Gamma}\left(-\nabla u^{k}(s)+X^{k}(s)\right) \cdot \nabla \dot{w}(s) \mathrm{d} x \mathrm{~d} s,
\end{aligned}
$$

where we defined

$$
u^{k}(s):=u\left(t_{k}^{j+1}\right) \quad \text { and } \quad X^{k}(s):=\frac{1}{2} \int_{t_{k}^{j}}^{t_{k}^{j+1}} \nabla \dot{w}(\tau) \mathrm{d} \tau
$$

for every $s \in\left(t_{k}^{j}, t_{k}^{j+1}\right]$. Notice that since $w \in H^{1}\left((0, T) ; H^{1}(\Omega)\right)$,

$$
\left\|X^{k}(s)\right\| \rightarrow 0 \text { uniformly with respect to } s \in[0, t] \text {. }
$$

Iterating the previous inequality with $j=n(k)-1, \ldots, 0$ we obtain

$$
E\left(u(t), \nu^{t}\right) \geq E\left(u(0), \nu^{0}\right)+\int_{0}^{t} \int_{\Omega \backslash \Gamma}\left(\nabla u^{k}(s)-X^{k}(s)\right) \cdot \nabla \dot{w}(s) \mathrm{d} x \mathrm{~d} s .
$$

Thanks to (3.7) we have that for a.e. $s \in[0, t]$

$$
\int_{\Omega \backslash \Gamma} X^{k}(s) \cdot \nabla \dot{w}(s) \mathrm{d} x \stackrel{k \rightarrow \infty}{\longrightarrow} 0 .
$$


Taking into account last relation and applying Lebesgue dominated convergence theorem

$$
\int_{0}^{t} \int_{\Omega \backslash \Gamma}\left(\nabla u^{k}(s)-X^{k}(s)\right) \cdot \nabla \dot{w}(s) \mathrm{d} x \mathrm{~d} s-\int_{0}^{t} \int_{\Omega \backslash \Gamma} \nabla u^{k}(s) \cdot \nabla \dot{w}(s) \mathrm{d} x \mathrm{~d} s \stackrel{k \rightarrow \infty}{\longrightarrow} 0,
$$

so that

$$
E\left(u(t), \nu^{t}\right)-E\left(u(0), \nu^{0}\right) \geq \limsup _{k \rightarrow+\infty} \int_{0}^{t} \int_{\Omega \backslash \Gamma} \nabla u^{k}(s) \cdot \nabla \dot{w}(s) \mathrm{d} x \mathrm{~d} s .
$$

In order to conclude the proof, let us recall a general result in measure theory (see [6], Lem. 4.12).

Lemma 3.27. Let $X$ be a Banach space and $f \in L^{1}((0, t) ; X)$. Then, there exists a sequence of subdivisions $0=t_{k}^{0} \leq t_{k}^{1} \leq \ldots \leq t_{k}^{n(k)-1} \leq t_{k}^{n(k)}=t$, satisfying (3.6), such that

$$
\lim _{k \rightarrow+\infty} \sum_{i=1}^{n(k)} \int_{t_{k}^{i-1}}^{t_{k}^{i}}\left\|f\left(t_{k}^{i}\right)-f(s)\right\|_{X} \mathrm{~d} s=0
$$

In particular, we have

and

$$
\lim _{k \rightarrow+\infty} \sum_{i=1}^{n(k)}\left\|\left(t_{k}^{i}-t_{k}^{i-1}\right) f\left(t_{k}^{i}\right)-\int_{t_{k}^{i-1}}^{t_{k}^{i}} f(s) \mathrm{d} s\right\|_{X}=0
$$

$$
\sum_{i=1}^{n(k)}\left(t_{k}^{i}-t_{k}^{i-1}\right) f\left(t_{k}^{i}\right) \longrightarrow \int_{0}^{t} f(s) \mathrm{d} s \quad \text { strongly in } X .
$$

We apply the previous lemma to the function

$$
f: s \mapsto\left(\int_{\Omega \backslash \Gamma} \nabla u(s) \cdot \nabla \dot{w}(s) \mathrm{d} x, \nabla \dot{w}(s)\right)
$$

with $X=\mathbb{R} \times L^{2}\left(\Omega ; \mathbb{R}^{N}\right)$.

Hence, there exists a sequence of subdivisions $0=t_{k}^{0} \leq t_{k}^{1} \leq \ldots \leq t_{k}^{n(k)-1} \leq t_{k}^{n(k)}=t$ such that

$$
\int_{0}^{t} \int_{\Omega \backslash \Gamma} \nabla u^{k}(s) \cdot W^{k}(s) \mathrm{d} x \mathrm{~d} s \longrightarrow \int_{0}^{t} \int_{\Omega \backslash \Gamma} \nabla u(s) \cdot \nabla \dot{w}(s) \mathrm{d} x \mathrm{~d} s,
$$

and

$$
\int_{0}^{t} \int_{\Omega \backslash \Gamma} \nabla u^{k}(s) \cdot\left(\nabla \dot{w}(s)-W^{k}(s)\right) \mathrm{d} x \mathrm{~d} s \rightarrow 0,
$$

where

$$
W^{k}(s):=\nabla \dot{w}\left(t_{k}^{i}\right), \quad t_{k}^{i-1}<s \leq t_{k}^{i}
$$

From (3.9), using (3.10) and (3.11), we get the thesis.

The next proposition gives a property of irreversible quasistatic evolutions whose internal variable is localized for $t=0$.

Proposition 3.28. Let $t \mapsto\left(u(t), \nu^{t}\right)$ be an irreversible quasistatic evolution and let $\nu^{0}=\delta_{\gamma_{0}}$, with $\gamma_{0}: \Gamma \rightarrow$ $[0,2 M]$ a measurable function. Let us define for every $t \in[0, T]$

$$
\gamma(t):=\gamma_{0} \vee \underset{0 \leq s \leq t}{\operatorname{ess} \sup _{0}}|[u(s)]|
$$


Then, there holds

$$
\delta_{\gamma(t)} \preceq \nu^{t} \quad \text { for every } t \in[0, T] .
$$

Proof. Let $t \in[0, T]$ be fixed. As $\left(u(s), \nu^{s}\right) \in a(s, w)$ for every $s \in[0, t]$, in particular we have that

$$
\delta_{|[u(s)]|} \preceq \nu^{s} \quad \text { for every } s \in[0, t] .
$$

Since $s \mapsto \nu^{s}$ is increasing and thanks to Remark 3.17, from the previous inequality we get that

$$
\delta_{\operatorname{ess} \sup _{0 \leq s \leq t}|[u(s)]|} \preceq \nu^{t} .
$$

Recalling that $\delta_{\gamma_{0}}=\nu^{0} \preceq \nu^{t}$ the thesis follows.

Remark 3.29. From the previous proposition it follows that for $\mathcal{H}^{N-1}$-a.e. $x \in \Gamma$ there holds

$$
\left(\nu^{t}\right)_{x}([0, \gamma(t)(x)))=0
$$

i.e. that $\operatorname{supp}\left(\nu^{t}\right)_{x} \subset[\gamma(t)(x), 2 M]$.

Finally, we state the main result of the paper.

Theorem 3.30. Let $w \in H^{1}\left((0, T) ; H^{1}(\Omega)\right)$ with $\sup _{t \in[0, T]}\|w(t)\|_{L^{\infty}(\Omega)}=M<+\infty$, and let $\left(u_{0}, \delta_{\gamma_{0}}\right)$ be globally stable at time $t=0$, where $\gamma_{0}: \Gamma \rightarrow[0,2 M]$ is a measurable function. Then there exists an irreversible quasistatic evolution in the sense of Young measures $t \mapsto\left(u(t), \nu^{t}\right)$ such that $\left(u(0), \nu^{0}\right)=\left(u_{0}, \delta_{\gamma_{0}}\right)$.

\section{Proof of Theorem 3.30}

In this section we prove the existence of an irreversible quasistatic evolution in the sense of Young measures, by means of a time discretization procedure. For every $k \in \mathbb{N}$, let us fix a collection of times $\left(t_{k}^{i}\right)_{0 \leq i \leq k}$ in $[0, T]$ such that $0=t_{k}^{0}<t_{k}^{1}<\ldots<t_{k}^{k-1}<t_{k}^{k}=T$ and

$$
\lim _{k \rightarrow+\infty} \max _{1 \leq i \leq k}\left(t_{k}^{i}-t_{k}^{i-1}\right)=0
$$

We set $w_{k}^{i}:=w\left(t_{k}^{i}\right)$ for $k \in \mathbb{N}$ and $i=0, \ldots, k$. For $k \in \mathbb{N}$ fixed and $i=0, \ldots, k$, we define the pair $\left(u_{k}^{i}, \gamma_{k}^{i}\right)$ in the following way. First, we set $\left(u_{k}^{0}, \gamma_{k}^{0}\right):=\left(u_{0}, \gamma_{0}\right)$. Now, let us assume that the pair $\left(u_{k}^{i-1}, \gamma_{k}^{i-1}\right)$ is given. By induction, we define $u_{k}^{i}$ as a solution of the problem

$$
\min \left\{E\left(v,|[v]| \vee \gamma_{k}^{i-1}\right): v \in H^{1}(\Omega \backslash \Gamma), v=w_{k}^{i} \text { on } \partial_{D} \Omega\right\}
$$

Then, we set $\gamma_{k}^{i}:=\gamma_{k}^{i-1} \vee\left|\left[u_{k}^{i}\right]\right|$. The existence of a solution of (4.1) can be proved by using the direct method of the Calculus of Variations.

Remark 4.1. Consider the minimum problem

$$
\min \left\{E(v, \tau):(v, \tau) \in \mathcal{A}\left(t_{k}^{i}, w\right), \tau \geq \gamma_{k}^{i-1}\right\}
$$

Arguing as in Remark 3.22, one can see that the following facts are equivalent:

- the pair $\left(u_{k}^{i}, \gamma_{k}^{i}\right)$ is a solution to (4.2);

- $u_{k}^{i}$ is a solution to $(4.1)$ and $\gamma_{k}^{i}=\gamma_{k}^{i-1} \vee\left|\left[u_{k}^{i}\right]\right|$. 
We define now

$$
u_{k}(t):=u_{k}^{i}, \quad \gamma_{k}(t):=\gamma_{k}^{i}, \quad w_{k}(t):=w\left(t_{k}^{i}\right) \quad \text { for } t_{k}^{i} \leq t<t_{k}^{i+1} .
$$

Notice that $t \mapsto \gamma_{k}(t)$ is nondecreasing, since $\gamma_{k}^{i-1} \leq \gamma_{k}^{i}$. By definition of $\left(u_{k}^{i}, \gamma_{k}^{i}\right)$ and from the fact that $\varphi(|[v]|, \cdot)$ is nondecreasing, we get that for $t_{k}^{i} \leq t<t_{k}^{i+1}$

$$
\frac{1}{2} \int_{\Omega \backslash \Gamma}\left|\nabla u_{k}(t)\right|^{2} \mathrm{~d} x+\int_{\Gamma} \varphi\left(\left|\left[u_{k}(t)\right]\right|, \gamma_{k}^{i}\right) \mathrm{d} x \leq \frac{1}{2} \int_{\Omega \backslash \Gamma}|\nabla v|^{2} \mathrm{~d} x+\int_{\Gamma} \varphi\left(|[v]|,|[v]| \vee \gamma_{k}^{i}\right) \mathrm{d} x
$$

for every $v \in H^{1}(\Omega \backslash \Gamma)$ with $v=w\left(t_{k}^{i}\right)$ on $\partial_{D} \Omega$. By Remark 2.3, we can assume

$$
\left\|\gamma_{k}(t)\right\|_{L^{\infty}(\Gamma)} \leq 2 M
$$

By Theorem 3.20, there exists a nondecreasing function $\nu:[0, T] \rightarrow \mathcal{Y}\left(\Gamma, \mathcal{H}^{N-1},[0,2 M]\right)$ and a subsequence $k_{j} \rightarrow+\infty$ such that for every $t \in[0, T]$

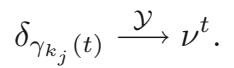

Let now $\Theta \subset[0, T]$ be such that $\mathcal{L}^{1}(\Theta)=0$ and $\dot{w}(t)$ is well defined for every $t \in[0, T] \backslash \Theta$. We set

$$
\theta_{k_{j}}(t):= \begin{cases}\int_{\Omega \backslash \Gamma} \nabla u_{k_{j}}(t) \cdot \nabla \dot{w}(t) \mathrm{d} x & \text { for } t \in[0, T] \backslash \Theta \\ 0 & \text { for } t \in \Theta\end{cases}
$$

By Remark 2.3 it follows that there exists a constant $C$, independent of $t$ and $j$, such that

$$
\left\|u_{k_{j}}(t)\right\|_{H^{1}(\Omega \backslash \Gamma)} \leq C .
$$

For every $t \in[0, T]$, we set

$$
\theta(t):=\limsup _{j \rightarrow+\infty} \theta_{k_{j}}(t) .
$$

Notice that $\theta$ is measurable, since it is the pointwise limsup of a countable family of measurable functions. Moreover,

$$
\int_{0}^{T} \theta(s) \mathrm{d} s \leq \int_{0}^{T} \limsup _{j \rightarrow+\infty}\left\|\nabla u_{k_{j}}(s)\right\|\|\nabla \dot{w}(s)\| \mathrm{d} s \leq C,
$$

so that $\theta \in L^{1}(0, T)$. By definition of $\theta$, for every $t \in[0, T]$ we can still extract a subsequence, possibly depending on $t$ and denoted by $\left(k_{j}(t)\right)_{j \in \mathbb{N}}$, such that

$$
\theta(t)=\lim _{j \rightarrow+\infty} \theta_{k_{j}(t)}(t)
$$

From (4.7) it follows that we can extract a further subsequence (not relabeled) such that for every $t \in[0, T]$

$$
u_{k_{j}(t)}(t) \rightarrow u(t) \text { weakly in } H^{1}(\Omega \backslash \Gamma)
$$

and

for some $u(t) \in H^{1}(\Omega \backslash \Gamma)$.

$$
\left[u_{k_{j}(t)}(t)\right] \rightarrow[u(t)] \quad \mathcal{H}^{N-1} \text {-a.e. on } \Gamma
$$

Finally, we observe that, thanks to (4.9), for every $t \in[0, T] \backslash \Theta$

$$
\theta(t)=\lim _{j \rightarrow+\infty} \int_{\Omega \backslash \Gamma} \nabla u_{k_{j}(t)}(t) \cdot \nabla \dot{w}(t) \mathrm{d} x=\int_{\Omega \backslash \Gamma} \nabla u(t) \cdot \nabla \dot{w}(t) \mathrm{d} x .
$$


We prove now that the function $t \mapsto\left(u(t), \nu^{t}\right)$ from $[0, T]$ to $H^{1}(\Omega \backslash \Gamma) \times \mathcal{Y}\left(\Gamma, \mathcal{H}^{N-1},[0,2 M]\right)$ is an irreversible quasistatic evolution. We begin by showing the global stability property $\left(\mathrm{a}^{\prime}\right)$.

Lemma 4.2. For every $t \in[0, T]$ we have that $\left(u(t), \nu^{t}\right) \in a(t, w)$ and

$$
\begin{aligned}
\frac{1}{2} \int_{\Omega \backslash \Gamma}|\nabla u(t)|^{2} \mathrm{~d} x+\int_{\Gamma} \int_{[0,2 M]} \varphi(|[u(t)]|, \xi) \mathrm{d} \nu_{x}^{t}(\xi) \mathrm{d} \mathcal{H}^{N-1} \leq & \frac{1}{2} \int_{\Omega \backslash \Gamma}|\nabla v|^{2} \mathrm{~d} x \\
& +\int_{\Gamma} \int_{[0,2 M]} \varphi(|[v]|,|[v]| \vee \xi) \mathrm{d} \nu_{x}^{t}(\xi) \mathrm{d} \mathcal{H}^{N-1}
\end{aligned}
$$

for every $v \in H^{1}(\Omega \backslash \Gamma)$ with $v=w(t)$ on $\partial_{D} \Omega$.

Proof. Fix $t \in[0, T]$. By (4.9) and from the fact that $w_{k_{j}(t)}(t) \rightarrow w(t)$ strongly in $H^{1}(\Omega)$ it follows that $u(t) \in H^{1}(\Omega \backslash \Gamma)$ and $u(t)=w(t)$ on $\partial_{D} \Omega$. Moreover, by definition of $\gamma_{k}$, we have that

$$
\delta_{\left|\left[u_{k_{j}(t)}(t)\right]\right|} \preceq \delta_{\gamma_{k_{j}(t)}(t)} .
$$

Passing to the limit as $j \rightarrow+\infty$, thanks to (4.6), (4.10) and Remark 3.14, we get

$$
\delta_{|[u(t)]|} \preceq \nu^{t},
$$

and hence $\left(u(t), \nu^{t}\right) \in a(t, w)$.

To prove (4.12), let $v \in H^{1}(\Omega \backslash \Gamma)$ with $v=w(t)$ on $\partial_{D} \Omega$ and set $v_{k_{j}(t)}:=v-w(t)+w_{k_{j}(t)}(t)$. By (4.4) we get

$$
\begin{aligned}
\frac{1}{2} \int_{\Omega \backslash \Gamma}\left|\nabla u_{k_{j}(t)}(t)\right|^{2} \mathrm{~d} x+\int_{\Gamma} \varphi\left(\left|\left[u_{k_{j}(t)}(t)\right]\right|, \gamma_{k_{j}(t)}(t)\right) \mathrm{d} \mathcal{H}^{N-1} \leq \frac{1}{2} \int_{\Omega \backslash \Gamma}\left|\nabla v_{k_{j}(t)}\right|^{2} \mathrm{~d} x & \\
& +\int_{\Gamma} \varphi\left(|[v]|,|[v]| \vee \gamma_{k_{j}(t)}(t)\right) \mathrm{d} \mathcal{H}^{N-1}
\end{aligned}
$$

Since $v_{k_{j}(t)} \rightarrow v$ strongly in $H^{1}(\Omega \backslash \Gamma)$, using Theorem 3.8 and the fact that $\varphi$ is bounded we can pass to the limit as $j \rightarrow+\infty$ in the right-hand side of (4.13), obtaining the right-hand side of (4.12). Thanks to (4.9), to prove (4.12) it remains to show that

$$
\int_{\Gamma} \varphi\left(\left|\left[u_{k_{j}(t)}(t)\right]\right|, \gamma_{k_{j}(t)}(t)\right) \mathrm{d} \mathcal{H}^{N-1} \rightarrow \int_{\Gamma} \int_{[0,2 M]} \varphi(|[u(t)]|, \xi) \mathrm{d} \nu_{x}^{t}(\xi) \mathrm{d} \mathcal{H}^{N-1}
$$

To this purpose, it will be useful to consider the following extension of $\varphi$ :

$$
\tilde{\varphi}(y, z):= \begin{cases}\varphi(y, z) & \text { for } 0 \leq y \leq z ; \\ g(z) & \text { for } 0 \leq z<y .\end{cases}
$$

Then, using again Theorem 3.8

$$
\int_{\Gamma} \tilde{\varphi}\left(|[u](t)|, \gamma_{k_{j}(t)}(t)\right) \mathrm{d} \mathcal{H}^{N-1} \rightarrow \int_{\Gamma} \int_{[0,2 M]} \tilde{\varphi}(|[u](t)|, \xi) \mathrm{d} \nu_{x}^{t}(\xi) \mathrm{d} \mathcal{H}^{N-1}=\int_{\Gamma} \int_{[0,2 M]} \varphi(|[u](t)|, \xi) \mathrm{d} \nu_{x}^{t}(\xi) \mathrm{d} \mathcal{H}^{N-1}
$$

Thus, it remains to prove that

$$
I_{j}:=\int_{\Gamma}\left(\tilde{\varphi}\left(|[u(t)]|, \gamma_{k_{j}(t)}(t)\right)-\varphi\left(\left|\left[u_{k_{j}(t)}(t)\right]\right|, \gamma_{k_{j}(t)}(t)\right)\right) \mathrm{d} \mathcal{H}^{N-1} \longrightarrow 0 .
$$


We have

$$
\begin{aligned}
\left|I_{j}\right| \leq & \int_{\Gamma \cap\left\{\gamma_{k_{j}(t)}(t) \geq|[u(t)]|\right\}}\left|\varphi\left(|[u(t)]|, \gamma_{k_{j}(t)}(t)\right)-\varphi\left(\left|\left[u_{k_{j}(t)}(t)\right]\right|, \gamma_{k_{j}(t)}(t)\right)\right| \mathrm{d} \mathcal{H}^{N-1} \\
& +\int_{\Gamma \cap\left\{\gamma_{k_{j}(t)}(t)<|[u(t)]|\right\}}\left|\varphi\left(\gamma_{k_{j}(t)}(t), \gamma_{k_{j}(t)}(t)\right)-\varphi\left(\left|\left[u_{k_{j}(t)}(t)\right]\right|, \gamma_{k_{j}(t)}(t)\right)\right| \mathrm{d} \mathcal{H}^{N-1}
\end{aligned}
$$

Since $\varphi(\cdot, z)$ is Lipschitz in $[0, z]$, with Lipschitz constant $L$ independent of $z$,

$$
\begin{aligned}
\left|I_{j}\right| \leq & L \int_{\Gamma \cap\left\{\gamma_{k_{j}(t)}(t) \geq|[u(t)]|\right\}}||[u(t)]|-|\left[u_{k_{j}(t)}(t)\right]|| \mathrm{d} \mathcal{H}^{N-1} \\
& +L \int_{\Gamma \cap\left\{\gamma_{k_{j}(t)}(t)<|[u(t)]|\right\}}\left(\gamma_{k_{j}(t)}(t)-\left|\left[u_{k_{j}(t)}(t)\right]\right|\right) \mathrm{d} \mathcal{H}^{N-1} \\
\leq & L \int_{\Gamma \cap\left\{\gamma_{k_{j}(t)}(t) \geq|[u(t)]|\right\}}||[u(t)]|-|\left[u_{k_{j}(t)}(t)\right]|| \mathrm{d} \mathcal{H}^{N-1} \\
& +L \int_{\Gamma \cap\left\{\gamma_{k_{j}(t)}(t)<|[u(t)]|\right\}}\left(|[u(t)]|-\left|\left[u_{k_{j}(t)}(t)\right]\right|\right) \mathrm{d} \mathcal{H}^{N-1} \\
= & L \int_{\Gamma}||[u(t)]|-|\left[u_{k_{j}(t)}(t)\right]|| \mathrm{d} \mathcal{H}^{N-1} .
\end{aligned}
$$

Passing to the limit as $j \rightarrow+\infty$ by (4.10) the proof is concluded.

The irreversibility condition $\left(b^{\prime}\right)$ follows by construction, as a consequence of Helly's selection principle (Thm. 3.20). In order to prove the energy equality, we first give an energy estimate for the discrete time evolutions in terms of $\theta_{k}$.

Lemma 4.3. There exists a numerical sequence $R_{k} \rightarrow 0$ such that

$$
E\left(u_{k}^{i}, \gamma_{k}^{i}\right) \leq E\left(u_{0}, \gamma_{0}\right)+\int_{0}^{t_{k}^{i}} \theta_{k}(s) \mathrm{d} s+R_{k}
$$

for any $k \in \mathbb{N}$ and for any $i=1, \ldots, k$.

Proof. Fix $k \in \mathbb{N}$ and $i \in\{1, \ldots, k\}$. Let $j \in \mathbb{N}$ be such that $1 \leq j \leq i$. By the minimality of $\left(u_{k}^{j}, \gamma_{k}^{j}\right)$, choosing $\left(u_{k}^{j-1}-w_{k}^{j-1}+w_{k}^{j}, \gamma_{k}^{j-1}\right)$ as test pair we have

$$
\begin{aligned}
\frac{1}{2} \int_{\Omega \backslash \Gamma}\left|\nabla u_{k}^{j}\right|^{2} \mathrm{~d} x+\int_{\Gamma} \varphi\left(\left|\left[u_{k}^{j}\right]\right|, \gamma_{k}^{j}\right) \mathrm{d} \mathcal{H}^{N-1} \leq & \frac{1}{2} \int_{\Omega \backslash \Gamma}\left|\nabla u_{k}^{j-1}\right|^{2} \mathrm{~d} x+\int_{\Gamma} \varphi\left(\left|\left[u_{k}^{j-1}\right]\right|, \gamma_{k}^{j-1}\right) \mathrm{d} \mathcal{H}^{N-1} \\
& +\int_{t_{k}^{j-1}}^{t_{k}^{j}} \int_{\Omega \backslash \Gamma} \nabla u_{k}(s) \cdot \nabla \dot{w}(s) \mathrm{d} x \mathrm{~d} s+\frac{1}{2}\left(\int_{t_{k}^{j-1}}^{t_{k}^{j}}\|\nabla \dot{w}(s)\| \mathrm{d} s\right)^{2} \\
\leq & \frac{1}{2} \int_{\Omega \backslash \Gamma}\left|\nabla u_{k}^{j-1}\right|^{2} \mathrm{~d} x+\int_{\Gamma} \varphi\left(\left|\left[u_{k}^{j-1}\right]\right|, \gamma_{k}^{j-1}\right) \mathrm{d} x \\
& +\int_{t_{k}^{j-1}}^{t_{k}^{j}} \int_{\Omega \backslash \Gamma} \nabla u_{k}(s) \cdot \nabla \dot{w}(s) \mathrm{d} x \mathrm{~d} s \\
& +\frac{1}{2} \max _{r=1, \ldots, k}\left(\int_{t_{k}^{r-1}}^{t_{k}^{r}}\|\nabla \dot{w}(s)\| \mathrm{d} s\right) \int_{t_{k}^{j-1}}^{t_{k}^{j}}\|\nabla \dot{w}(s)\| \mathrm{d} s .
\end{aligned}
$$


Iterating the previous inequality for $j=i, \ldots, 1$, we get the thesis with

$$
R_{k}=\frac{1}{2} \max _{r=1, \ldots, k}\left(\int_{t_{k}^{r-1}}^{t_{k}^{r}}\|\nabla \dot{w}(s)\| \mathrm{d} s\right) \int_{0}^{T}\|\nabla \dot{w}(s)\| \mathrm{d} s
$$

that goes to 0 as $k \rightarrow+\infty$ because of the absolute continuity of the integral.

Using the previous lemma, we prove the energy balance condition (c) for the continuous-time evolution.

Lemma 4.4. For every $t \in[0, T]$ we have

$$
E\left(u(t), \nu^{t}\right)=E\left(u_{0}, \gamma_{0}\right)+\int_{0}^{t} \theta(s) \mathrm{d} s .
$$

Proof. By (4.8), we already know that $\theta \in L^{1}([0, T])$. Let $t \in[0, T]$ be fixed, and let $i$ be such that $t_{k_{j}(t)}^{i} \leq t<$ $t_{k_{j}(t)}^{i+1}$. Applying the previous lemma, we have

$$
E\left(u_{k_{j}(t)}(t), \gamma_{k_{j}(t)}(t)\right) \leq E\left(u_{0}, \gamma_{0}\right)+\int_{0}^{t_{k_{j}(t)}^{i}} \theta_{k_{j}(t)}(s) \mathrm{d} s+R_{k_{j}(t)}
$$

Recalling that $k_{j}(t)$ is a subsequence of $k_{j}$, and that $k_{j}$ does not depend on $t$, we get

$$
\begin{aligned}
E\left(u(t), \nu^{t}\right) & \leq \liminf _{j \rightarrow+\infty} E\left(u_{k_{j}(t)}(t), \gamma_{k_{j}(t)}(t)\right) \leq E\left(u_{0}, \gamma_{0}\right)+\limsup _{j \rightarrow+\infty} \int_{0}^{t_{k_{j}(t)}^{i}} \theta_{k_{j}(t)}(s) \mathrm{d} s \\
& \leq E\left(u_{0}, \gamma_{0}\right)+\int_{0}^{t} \limsup _{j \rightarrow+\infty} \theta_{k_{j}}(s) \mathrm{d} s=E\left(u_{0}, \gamma_{0}\right)+\int_{0}^{t} \theta(s) \mathrm{d} s,
\end{aligned}
$$

where we used (4.9), (4.14) and Fatou's lemma. The proof is concluded, observing that the opposite energy inequality comes from Theorem 3.26 .

\section{The CASE OF CONSTANT UnlOADING RESPONSE}

In this section we show that in the particular situation in which the function $\varphi$ does not depend on the jump (i.e. $\varphi$ is constant with respect to the first variable), the irreversible quasistatic evolution $t \mapsto\left(u(t), \nu^{t}\right)$ provided by Theorem 3.30 is such that $\nu^{t}=\delta_{\gamma(t)}$ for every $t \in[0, T]$, where $\gamma(t)$ is defined in (3.12). In this way, we recover the result that Dal Maso and Zanini proved by means of a suitable notion of convergence in [5]. In addition, we show that the discrete-time internal variables $\gamma_{k}$ defined in (4.3) actually converge in measure to $\gamma(t)$. This improves the $\sigma$-convergence result stated in [5].

Theorem 5.1. Let the hypotheses of Theorem 3.30 be satisfied. Assume in addition that $\varphi(\cdot, z)$ is constant for every $z \in[0,+\infty)$ and that $\varphi(y, \cdot)$ is strictly increasing for every $y \in[0,+\infty)$. Then, the function $t \mapsto\left(u(t), \nu^{t}\right)$ provided by Theorem 3.30 is such that:

$-\nu^{t}=\delta_{\gamma(t)}$ for every $t \in[0, T]$, where $\gamma(t)$ is defined in (3.12);

- $t \mapsto(u(t), \gamma(t))$ is an irreversible quasistatic evolution.

Remark 5.2. It follows from Theorem 5.1 and Proposition 3.9 that when $\varphi(\cdot, z)$ is constant for every $z \in$ $[0,+\infty)$ and $\varphi(y, \cdot)$ is strictly increasing for every $y \in[0,+\infty)$

$$
\gamma_{k_{j}(t)}(t) \longrightarrow \gamma(t) \text { in measure on } \Gamma \text {. }
$$


Remark 5.3. If one removes the assumption that $\varphi(y, \cdot)$ is strictly increasing, in general the equality $\nu^{t}=\delta_{\gamma(t)}$ does not hold. Nevertheless, we have that the function $t \mapsto(u(t), \gamma(t))$ is an irreversible quasistatic evolution with the property $E\left(u(t), \nu^{t}\right)=E\left(u(t), \delta_{\gamma(t)}\right)$ for every $t \in[0, T]$.

Proof. By Theorem 3.30, there exists an irreversible quasistatic evolution in the sense of Young measures $t \mapsto\left(u(t), \nu^{t}\right)$ such that $\left(u(0), \nu^{0}\right)=\left(u_{0}, \delta_{\gamma_{0}}\right)$. Let us show that $\nu^{t}=\delta_{\gamma(t)}$ for every $t \in(0, T]$. Let us fix $t \in(0, T]$. Since $\left(u(t), \nu^{t}\right)$ is globally stable, for every $v \in H^{1}(\Omega \backslash \Gamma)$ with $v=w(t)$ on $\partial_{D} \Omega$ we have

$\frac{1}{2} \int_{\Omega \backslash \Gamma}|\nabla u(t)|^{2} \mathrm{~d} x \leq \frac{1}{2} \int_{\Omega \backslash \Gamma}|\nabla v|^{2} \mathrm{~d} x+\int_{\Gamma} \int_{[0,2 M]} \varphi(|[v]|,|[v]| \vee \xi) \mathrm{d} \nu_{x}^{t}(\xi) \mathrm{d} \mathcal{H}^{N-1}$

$$
-\int_{\Gamma} \int_{[0,2 M]} \varphi(|[u(t)]|, \xi) \mathrm{d} \nu_{x}^{t}(\xi) \mathrm{d} \mathcal{H}^{N-1} .
$$

Using the fact that $\varphi(\cdot, z)$ is constant for every $z \in[0,+\infty)$, we can write

$$
\begin{aligned}
\int_{\Gamma} \int_{[0,2 M]}(\varphi(|[v]|,|[v]| \vee \xi)-\varphi(|[u(t)]|, \xi)) \mathrm{d} \nu_{x}^{t}(\xi) \mathrm{d} \mathcal{H}^{N-1} & \\
& =\int_{\Gamma} \int_{\{\xi<|[v]|\}}(\varphi(|[v]|,|[v]|)-\varphi(|[u(t)]|, \xi)) \mathrm{d} \nu_{x}^{t}(\xi) \mathrm{d} \mathcal{H}^{N-1} .
\end{aligned}
$$

Since, by Remark 3.29, $\gamma(t) \leq \xi$ for every $\xi \in \operatorname{supp}\left(\nu_{x}^{t}\right)$, and $\varphi(y, \cdot)$ is increasing for every $y \in[0,+\infty)$, we have

$$
\begin{aligned}
\int_{\Gamma} \int_{\{\xi<|[v]|\}}(\varphi(|[v]|,|[v]|) & -\varphi(|[u(t)]|, \xi)) \mathrm{d} \nu_{x}^{t}(\xi) \mathrm{d} \mathcal{H}^{N-1} \\
& \leq \int_{\Gamma} \int_{\{\xi<|[v]|\}}(\varphi(|[v]|,|[v]|)-\varphi(|[u(t)]|, \gamma(t))) \mathrm{d} \nu_{x}^{t}(\xi) \mathrm{d} \mathcal{H}^{N-1} \\
& =\int_{\Gamma} \int_{\{\xi<|[v]|\}}(\varphi(|[v]|,|[v]| \vee \gamma(t))-\varphi(|[u(t)]|, \gamma(t))) \mathrm{d} \nu_{x}^{t}(\xi) \mathrm{d} \mathcal{H}^{N-1} \\
& \leq \int_{\Gamma}(\varphi(|[v]|,|[v]| \vee \gamma(t))-\varphi(|[u(t)]|, \gamma(t))) \mathrm{d} \mathcal{H}^{N-1},
\end{aligned}
$$

where in the last inequality we used the fact that $\nu_{x}^{t}$ is a probability measure. Relations (5.1), (5.2) and (5.3) imply that

$$
\frac{1}{2} \int_{\Omega \backslash \Gamma}|\nabla u(t)|^{2} \mathrm{~d} x+\int_{\Gamma} \varphi(|[u(t)]|, \gamma(t)) \mathrm{d} \mathcal{H}^{N-1} \leq \frac{1}{2} \int_{\Omega \backslash \Gamma}|\nabla v|^{2} \mathrm{~d} x+\int_{\Gamma} \varphi(|[v]|,|[v]| \vee \gamma(t)) \mathrm{d} \mathcal{H}^{N-1},
$$

that is, by Remark 3.22, the pair $\left(u(t), \delta_{\gamma(t)}\right)$ is globally stable in the sense of Young measures at time $t$. Moreover, $t \mapsto \gamma(t)$ is nondecreasing. Hence, both conditions $\left(\mathrm{a}^{\prime}\right)$ and $\left(\mathrm{b}^{\prime}\right)$ of Definition 3.23 are fulfilled. We can then apply Theorem 3.26 to the function $t \mapsto\left(u(t), \delta_{\gamma(t)}\right)$. We get that for every $t \in[0, T]$

$$
E(u(t), \gamma(t)) \geq E\left(u_{0}, \gamma_{0}\right)+\int_{0}^{t} \int_{\Omega \backslash \Gamma} \nabla u(s) \cdot \nabla \dot{w}(s) \mathrm{d} x \mathrm{~d} s .
$$

Since $t \mapsto\left(u(t), \nu^{t}\right)$ is an irreversible quasistatic evolution in the sense of Young measures, from the last inequality it follows that $E(u(t), \gamma(t)) \geq E\left(u(t), \nu^{t}\right)$ for every $t \in[0, T]$. Hence, we have

$$
\int_{\Gamma} \int_{[0,2 M]} \varphi(|[u(t)]|, \gamma(t)) \mathrm{d} \nu_{x}^{t}(\xi) \mathrm{d} \mathcal{H}^{N-1} \geq \int_{\Gamma} \int_{[0,2 M]} \varphi(|[u(t)]|, \xi) \mathrm{d} \nu_{x}^{t}(\xi) \mathrm{d} \mathcal{H}^{N-1}
$$


for every $t \in[0, T]$. By Remark 3.29 and from the fact that $\varphi(|[u(t)]|, \cdot)$ is strictly increasing we get

$$
\nu^{t}=\delta_{\gamma(t)} \quad \text { for every } t \in[0, T] .
$$

\section{Concentration of the Measure $\nu^{t}$}

In Section 4 we provided an existence theorem for an irreversible quasistatic evolution $t \mapsto\left(u(t), \nu^{t}\right)$ in the sense of Young measures. To this aim, for every $k \in \mathbb{N}$ and for every $i=0,1, \ldots, k$ we defined by induction the pairs $\left(u_{k}^{i}, \gamma_{k}^{i}\right)$ with the property that

(i) $u_{k}^{i}$ is a solution to (4.1) and $\gamma_{k}^{i}=\left|\left[u_{k}^{i}\right]\right| \vee \gamma_{k}^{i-1}$.

Then, the piecewise constant functions $u_{k}:[0, T] \rightarrow H^{1}(\Omega \backslash \Gamma)$ and $\gamma_{k}:[0, T] \rightarrow L^{\infty}(\Gamma)^{+}$were defined in (4.3), and we proved that

(ii) $\left\|\left[u_{k}(t)\right]\right\|_{H^{\frac{1}{2}(\Gamma)}} \leq C$ for every $k \in \mathbb{N}, t \in[0, T]$;

(iii) $\gamma_{k_{j}}(t) \stackrel{\mathcal{Y}}{\longrightarrow} \nu^{t}$.

Moreover, we showed that in the particular case in which the unloading response $\varphi(\cdot, z)$ is constant, the limit measure $\nu^{t}$ has the special form $\nu^{t}=\delta_{\gamma(t)}$, where $\gamma(t) \in L^{\infty}(\Gamma)^{+}$for every $t \in[0, T]$ (Thm. 5.1).

In this section we investigate whether the same conclusion holds when $\varphi$ satisfies the hypotheses listed in Section 2, without further restrictions. In particular, we want to know if properties (i), (ii) and (iii) imply that $\nu^{t}$ is an atomic measure for every $t \in[0, T]$.

At the moment, we are not able to give a complete answer to this question. Nevertheless, we show with the following proposition that conditions (ii) and (iii) are not sufficient.

Proposition 6.1. There exists a sequence of nonnegative piecewise constant functions

$$
p_{k}:[0,1] \rightarrow H^{\frac{1}{2}}([0,2 \pi]) \quad k \in \mathbb{N},
$$

and a function

$$
\nu:[0,1] \rightarrow \mathcal{Y}\left([0,2 \pi], \mathcal{H}^{N-1},[0,1]\right)
$$

such that $\nu^{t}$ is non atomic for $t \in(0,1]$ and the following two conditions are satisfied:

(1) there exists a positive constant $C$ such that

$$
\left\|p_{k}(t)\right\|_{H^{\frac{1}{2}([0,2 \pi])}} \leq C,
$$

for every $t \in[0,1]$ and $k \in \mathbb{N}$;

(2) setting $\gamma_{k}(t):=\sup _{0 \leq s \leq t} p_{k}(s)$ there holds

$$
\gamma_{k}(t) \stackrel{\mathcal{Y}}{\longrightarrow} \nu^{t} \quad \text { for every } t \in[0,1] .
$$

Proof. Let $k \in \mathbb{N}$ be fixed. We set $p_{k}(t):=0$ for $0 \leq t<\frac{1}{2^{k}+1}$. When $\frac{2^{k}}{2^{k}+1} \leq t \leq 1$, we define

$$
p_{k}(t):= \begin{cases}\left(\sin \left(2^{k} x\right)\right)^{+} & \text {for } x \in\left[\frac{2 \pi\left(2^{k}-1\right)}{2^{k}}, 2 \pi\right], \\ 0 & \text { otherwise in }[0,2 \pi] .\end{cases}
$$

Let now $t \in(0,1)$ and let $i \in\left\{1, \ldots, 2^{k}-1\right\}$ be such that $\frac{i}{2^{k}+1} \leq t<\frac{i+1}{2^{k}+1}$. We assign $p_{k}(t)$ as

$$
p_{k}(t):= \begin{cases}\left(\sin \left(2^{k} x\right)\right)^{+} & \text {for } x \in\left[\frac{2 \pi(i-1)}{2^{k}}, \frac{2 \pi i}{2^{k}}\right], \\ 0 & \text { otherwise in }[0,2 \pi] .\end{cases}
$$


Notice that $p_{k}(t) \rightarrow 0$ strongly in $L^{2}([0,2 \pi])$ for every $t \in[0,1]$. Indeed,

$$
\left\|p_{k}(t)\right\|_{L^{2}([0,2 \pi])}^{2}=\int_{0}^{2 \pi}\left|p_{k}(t)\right|^{2} \mathrm{~d} x \leq \frac{\pi}{2^{k}} \rightarrow 0 .
$$

A standard computation shows that

$$
\left\|p_{k}(t)\right\|_{H^{\frac{1}{2}([0,2 \pi])}}^{2}<\pi(\pi+5) .
$$

Let us write the expression of the functions $\gamma_{k}(t)$. It turns out that $\gamma_{k}(t)=0$ for $0 \leq t<\frac{1}{2^{k}+1}$, and $\gamma_{k}(t)=\left(\sin \left(2^{k} x\right)\right)^{+}$when $\frac{2^{k}}{2^{k}+1} \leq t \leq 1$. Fix $t \in\left[\frac{1}{2^{k}+1}, \frac{2^{k}}{2^{k}+1}\right)$ and $i \in\left\{1, \ldots, 2^{k}-1\right\}$ such that $\frac{i}{2^{k}+1} \leq t<\frac{i+1}{2^{k}+1}$. Then

$$
\gamma_{k}(t)= \begin{cases}\left(\sin \left(2^{k} x\right)\right)^{+} & \text {for } x \in\left[0, \frac{2 \pi i}{2^{k}}\right] \\ 0 & \text { otherwise in }[0,2 \pi] .\end{cases}
$$

We will prove that for every $t \in[0,1]$ we have $\gamma_{k}(t) \stackrel{\mathcal{Y}}{\longrightarrow} \nu^{t}$, where $\nu^{0}=\delta_{0}$ and for $t \in(0,1]$

$$
\nu_{x}^{t}(\xi):=1_{[0,2 \pi t]}(x)\left(\frac{\pi}{\sqrt{1-\xi^{2}}} \mathrm{~d} \xi+\frac{1}{2} \delta_{0}(\xi)\right)+1_{(2 \pi t, 2 \pi]}(x) \delta_{0}(\xi)
$$

for $\mathcal{H}^{N-1}$-a.e. $x \in[0,2 \pi]$. To this purpose, it will be enough to show that

$$
\int_{0}^{2 \pi} a(x) f\left(p_{k}(t)(x)\right) \mathrm{d} x \longrightarrow \int_{0}^{2 \pi} \int_{0}^{1} a(x) f(\xi) \mathrm{d} x \mathrm{~d} \nu_{x}^{t}(\xi)
$$

for every $a \in C^{0}([0,2 \pi])$ and $f \in C^{0}([0,1])$. For $t=0$ the claim follows immediately. Let us fix $t \in(0,1)$, the proof for the case $t=1$ being analogous. For every $k \in \mathbb{N}$, we have that $\frac{i_{k}}{2^{k}+1} \leq t<\frac{i_{k}+1}{2^{k}+1}$, for some $i_{k} \in$ $\left\{0,1, \ldots, 2^{k}\right\}$. Let us fix $\varepsilon>0$ arbitrarily small. There exist two integers $j$ and $r_{j}$ such that $0 \leq 2 \pi t-\frac{2 \pi r_{j}}{2^{j}}<\varepsilon$. We have

$$
\int_{0}^{2 \pi} a(x) f\left(p_{k}(t)(x)\right) \mathrm{d} x=I_{k}^{1}+I_{k}^{2}
$$

where

We observe that

$$
I_{k}^{1}:=\int_{0}^{\frac{2 \pi i_{k}}{2^{k}}} a(x) f\left(\left(\sin \left(2^{k} x\right)\right)^{+}\right) \mathrm{d} x \quad \text { and } \quad I_{k}^{2}:=\int_{\frac{2 \pi i_{k}}{2^{k}}}^{2 \pi} a(x) f(0) \mathrm{d} x .
$$

$$
I_{k}^{2} \longrightarrow \int_{2 \pi t}^{2 \pi} a(x) f(0) \mathrm{d} x=\int_{0}^{2 \pi} \int_{0}^{1} a(x) f(\xi) 1_{(2 \pi t, 2 \pi]}(x) \delta_{0}(\xi) \mathrm{d} x .
$$

It remains to compute the limit of $I_{k}^{1}$ as $k \rightarrow \infty$. We write

$$
I_{k}^{1}=\int_{0}^{\frac{2 \pi r_{j}}{2^{j}}} a(x) f\left(\left(\sin \left(2^{k} x\right)\right)^{+}\right) \mathrm{d} x+R_{j, k}
$$

where

$$
R_{j, k}:=\int_{\frac{2 \pi r_{j}}{2^{j}}}^{\frac{2 \pi i_{k}}{2^{k}}} a(x) f\left(\left(\sin \left(2^{k} x\right)\right)^{+}\right) \mathrm{d} x \leq\|f\|_{C^{0}([0,2 \pi])} \int_{\frac{2 \pi r_{j}}{2^{j}}}^{\frac{2 \pi i_{k}}{2^{k}}}|a(x)| \mathrm{d} x .
$$

From the last inequality it follows that for $\varepsilon$ fixed

$$
-\|f\|_{C^{0}([0,2 \pi])} \int_{\frac{2 \pi r_{j}}{2 j}}^{2 \pi t}|a(x)| \mathrm{d} x \leq \liminf _{k \rightarrow+\infty} R_{j, k} \leq \limsup _{k \rightarrow+\infty} R_{j, k} \leq\|f\|_{C^{0}([0,2 \pi])} \int_{\frac{2 \pi r_{j}}{2^{j}}}^{2 \pi t}|a(x)| \mathrm{d} x .
$$


Letting $\varepsilon$ tend to 0 all the terms in the last relation converge to 0 . Thus, we conclude that

$$
\lim _{k \rightarrow+\infty} I_{k}^{1}=\lim _{j \rightarrow+\infty} \lim _{k \rightarrow+\infty} \int_{0}^{\frac{2 \pi r_{j}}{2^{j}}} a(x) f\left(\left(\sin \left(2^{k} x\right)\right)^{+}\right) \mathrm{d} x .
$$

Let us compute the limit (6.4). For every $k>j$, we have that $f\left(\left(\sin \left(2^{k} x\right)\right)^{+}\right)$is periodic in $\left[0, \frac{2 \pi r_{j}}{2^{j}}\right]$. Hence, using the Riemann-Lebesgue lemma we have that when $k \rightarrow+\infty$

$$
f\left(\left(\sin \left(2^{k} x\right)\right)^{+}\right) \rightarrow \frac{2^{j}}{2 \pi r_{j}} \int_{0}^{\frac{2 \pi r_{j}}{2^{j}}} f\left(\left(\sin \left(2^{j} x\right)\right)^{+}\right) \mathrm{d} x \quad \text { weakly* in } L^{\infty}\left(0, \frac{2 \pi r_{j}}{2^{j}}\right) .
$$

Since

$$
\begin{aligned}
\frac{2^{j}}{2 \pi r_{j}} \int_{0}^{\frac{2 \pi r_{j}}{2 j}} f\left(\left(\sin \left(2^{j} x\right)\right)^{+}\right) \mathrm{d} x & =\frac{2^{j}}{2 \pi}\left(\int_{0}^{\frac{\pi}{2 j}} f\left(\sin \left(2^{j} x\right)\right) \mathrm{d} x+\frac{\pi}{2^{j}} f(0)\right) \\
& =\frac{1}{2 \pi} \int_{0}^{\pi} f(\sin x) \mathrm{d} x+\frac{1}{2} f(0)=\int_{0}^{1} f(\xi) \frac{1}{\pi \sqrt{1-\xi^{2}}} \mathrm{~d} \xi+\frac{1}{2} f(0),
\end{aligned}
$$

we have

$$
\lim _{k \rightarrow+\infty} \int_{0}^{\frac{2 \pi r_{j}}{2^{j}}} a(x) f\left(\left(\sin \left(2^{k} x\right)\right)^{+}\right) \mathrm{d} x=\int_{0}^{\frac{2 \pi r_{j}}{2^{j}}} \int_{0}^{1} a(x) f(\xi) \frac{1}{\pi \sqrt{1-\xi^{2}}} \mathrm{~d} \xi \mathrm{d} x+\int_{0}^{\frac{2 \pi r_{j}}{2 j}} \frac{1}{2} a(x) f(0) \mathrm{d} x .
$$

Collecting (6.1), (6.2), (6.4) and (6.5) we conclude the proof.

\section{Euler-Lagrange COnditions}

In this section we study in detail the Euler-Lagrange conditions satisfied by a pair $(u, \nu)$ which is globally stable in the sense of Young measures at a fixed time $t \in[0, T]$.

Proposition 7.1. Let $t \in[0, T]$ be fixed and let $(u, \nu)$ be globally stable in the sense of Young measures at time t. For $x \in \Gamma$ define $\alpha(x):=\inf \left\{z: z \in \operatorname{supp} \nu_{x}\right\}$. Then

$$
\begin{aligned}
\int_{\Omega \backslash \Gamma} \nabla u \cdot \nabla \psi \mathrm{d} x & +\int_{\Gamma}|[\psi]| 1_{\{0=|[u]|=\alpha\}}\left(\sigma \nu_{x}(\{0\})+\int_{(0,2 M]} \frac{\partial \varphi}{\partial y}\left(0^{+}, \xi\right) \mathrm{d} \nu_{x}(\xi)\right) \mathrm{d} \mathcal{H}^{N-1} \\
& +\int_{\Gamma}|[\psi]| 1_{\{0=|[u]|<\alpha\}} \int_{[0,2 M]} \frac{\partial \varphi}{\partial y}\left(0^{+}, \xi\right) \mathrm{d} \nu_{x}(\xi) \mathrm{d} \mathcal{H}^{N-1} \\
& +\int_{\Gamma}[\psi] \operatorname{sgn}[u] 1_{\{0<|[u]|<\alpha\}} \int_{[0,2 M]} \frac{\partial \varphi}{\partial y}(|[u]|, \xi) \mathrm{d} \nu_{x}(\xi) \mathrm{d} \mathcal{H}^{N-1} \\
& +\int_{\Gamma}|[\psi]| 1_{\{0<[u][\psi]=\alpha|[\psi]|\}}\left(g^{\prime}(\alpha) \nu_{x}(\{\alpha\})+\int_{(\alpha, 2 M]} \frac{\partial \varphi}{\partial y}(\alpha, \xi) \mathrm{d} \nu_{x}(\xi)\right) \mathrm{d} \mathcal{H}^{N-1} \\
& -\int_{\Gamma}|[\psi]| 1_{\{0>[u][\psi]=-\alpha|[\psi]|\}} \int_{[0,2 M]} \frac{\partial \varphi}{\partial y}(\alpha, \xi) \mathrm{d} \nu_{x}(\xi) \mathrm{d} \mathcal{H}^{N-1} \geq 0
\end{aligned}
$$

for every $\psi \in H_{0}^{1}\left(\Omega \backslash \Gamma, \partial_{D} \Omega\right)$. 
Proof. Since $(u, \nu)$ is globally stable, thanks to Remark 3.22 we have that $E(u, \nu) \leq E\left(v, \nu \vee \delta_{|[v]|}\right)$ for every $v \in H^{1}(\Omega \backslash \Gamma)$ such that $v=w(t)$ on $\partial_{D} \Omega$. Thus, for every $\psi \in H_{0}^{1}\left(\Omega \backslash \Gamma, \partial_{D} \Omega\right)$ there holds

$$
\liminf _{\eta \rightarrow 0^{+}} \frac{E\left(u+\eta \psi, \nu \vee \delta_{|[u]+\eta[\psi]|}\right)-E(u, \nu)}{\eta} \geq 0
$$

Let us show that the liminf in (7.2) is actually a limit and that it coincides with the left-hand side of (7.1). First, for the volume part of the energy, it is clear that

$$
\lim _{\eta \rightarrow 0^{+}} \frac{1}{2} \int_{\Omega \backslash \Gamma} \frac{|\nabla u+\eta \nabla \psi|^{2}-|\nabla u|^{2}}{\eta} \mathrm{d} x=\int_{\Omega \backslash \Gamma} \nabla u \cdot \nabla \psi \mathrm{d} x .
$$

It remains to evaluate the limit:

$$
\lim _{\eta \rightarrow 0^{+}} \int_{\Gamma} \int_{[0,2 M]} \frac{\varphi(|[u]+\eta[\psi]|, \xi \vee(|[u]+\eta[\psi]|))-\varphi(|[u]|, \xi)}{\eta} \mathrm{d} \nu_{x}(\xi) \mathrm{d} \mathcal{H}^{N-1}
$$

To this aim, we notice that for $\mathcal{H}^{N-1}$-a.e. $x \in \Gamma$

$$
\alpha(x)=\max \left\{a \in[0,2 M]: \nu_{x}([a, 2 M])=1\right\},
$$

from which one can check that $\alpha$ is measurable and bounded. Hence, we can divide $\Gamma$ in the following four measurable subsets.

Step 1. $x \in\{0=|[u]|=\alpha\}$

Let $x \in\{0=|[u]|=\alpha\}$ be fixed. In this case, we have under the integral on $\Gamma$ the expression

$$
\begin{aligned}
& \int_{[0,2 M]} \frac{\varphi(\eta|[\psi](x)|, \xi \vee \eta|[\psi](x)|)-\varphi(0, \xi)}{\eta} \mathrm{d} \nu_{x}(\xi) \\
& \quad=\nu_{x}(\{0\}) \frac{g(\eta|[\psi](x)|)}{\eta}+\int_{(0,2 M]} \frac{\varphi(\eta|[\psi](x)|, \xi \vee \eta|[\psi](x)|)-\varphi(0, \xi)}{\eta} \mathrm{d} \nu_{x}(\xi)
\end{aligned}
$$

For $\eta$ sufficiently small and $\xi \in(0,2 M]$ fixed we can write

$$
\frac{\varphi(\eta|[\psi](x)|, \xi \vee \eta|[\psi](x)|)-\varphi(0, \xi)}{\eta}=\frac{\varphi(\eta|[\psi](x)|, \xi)-\varphi(0, \xi)}{\eta}=|[\psi](x)| \frac{\partial \varphi}{\partial y}(\beta, \xi),
$$

where $\beta \in(0, \eta|[\psi](x)|)$. Thus, applying Lebesgue dominated convergence theorem we get that

$$
\lim _{\eta \rightarrow 0^{+}} \int_{[0,2 M]} \frac{\varphi(\eta|[\psi](x)|, \xi \vee \eta|[\psi](x)|)-\varphi(0, \xi)}{\eta} \mathrm{d} \nu_{x}(\xi)=|[\psi](x)|\left(\sigma \nu_{x}(\{0\})+\int_{(0,2 M]} \frac{\partial \varphi}{\partial y}\left(0^{+}, \xi\right) \mathrm{d} \nu_{x}(\xi)\right) .
$$

Applying once again Lebesgue dominated convergence theorem we get the second term of the first line of (7.1). 
Step 2. $x \in\{0=|[u]|<\alpha\}$

For $x$ fixed and $\eta>0$ sufficiently small we have

$$
\begin{aligned}
\int_{[0,2 M]} \frac{\varphi(\eta|[\psi](x)|, \xi \vee \eta|[\psi](x)|)-\varphi(0, \xi)}{\eta} \mathrm{d} \nu_{x}(\xi) & =\int_{[0,2 M]} \frac{\varphi(\eta|[\psi](x)|, \xi)-\varphi(0, \xi)}{\eta} \mathrm{d} \nu_{x}(\xi) \\
& =\int_{[0,2 M]}|[\psi](x)| \frac{\partial \varphi}{\partial y}(\beta, \xi) \mathrm{d} \nu_{x}(\xi)
\end{aligned}
$$

with $\beta \in(0, \eta|[\psi](x)|)$. Applying twice Lebesgue dominated convergence theorem, first for the measure $\nu_{x}$, and then for $\mathcal{H}^{N-1}$, we obtain the second line of (7.1).

Step 3. $x \in\{0<|[u]|<\alpha\}$

For $x$ fixed and $\eta>0$ sufficiently small the integrand is given by

$$
\int_{[0,2 M]} \frac{\varphi(|[u](x)+\eta[\psi](x)|, \xi)-\varphi(|[u](x)|, \xi)}{\eta} \mathrm{d} \nu_{x}(\xi)
$$

Passing to the limit as $\eta \rightarrow 0^{+}$one gets

$$
\left.\int_{[0,2 M]}[\psi](x) \operatorname{sgn}([u](x)) \frac{\partial \varphi}{\partial y}(|[u](x)|, \xi)\right) \mathrm{d} \nu_{x}(\xi)
$$

This gives the third line of (7.1).

Step 4. $x \in\{0<|[u]|=\alpha\}$

Let us assume that $[u](x)[\psi](x)>0$. In this case we have $|[u](x)+\eta[\psi](x)|>|[u](x)|=\alpha(x)$. Hence,

$$
\begin{aligned}
\int_{[0,2 M]} \frac{\varphi(|[u](x)+\eta[\psi](x)|, \xi \vee(|[u](x)+\eta[\psi](x)|))-\varphi(\alpha(x), \xi)}{\eta} \mathrm{d} \nu_{x}(\xi) \\
=\nu_{x}(\{\alpha(x)\}) \frac{g(|[u](x)+\eta[\psi](x)|)-g(\alpha(x))}{\eta} \\
\quad+\int_{(\alpha(x), 2 M]} \frac{\varphi(|[u](x)+\eta[\psi](x)|, \xi \vee(|[u](x)+\eta[\psi](x)|))-\varphi(\alpha(x), \xi)}{\eta} \mathrm{d} \nu_{x}(\xi) .
\end{aligned}
$$

For $\xi \in(\alpha(x), 2 M]$ fixed and $\eta>0$ sufficiently small the ratio under the integral sign is given by

$$
\frac{\varphi(|[u](x)+\eta[\psi](x)|, \xi \vee(|[u](x)+\eta[\psi](x)|))-\varphi(\alpha(x), \xi)}{\eta}=\frac{\varphi(|[u](x)+\eta[\psi](x)|, \xi)-\varphi(\alpha(x), \xi)}{\eta}
$$

When $\eta \rightarrow 0^{+}$, the last expression becomes

$$
[\psi](x) \operatorname{sgn}([u](x)) \frac{\partial \varphi}{\partial y}(\alpha(x), \xi)=|[\psi](x)| \frac{\partial \varphi}{\partial y}(\alpha(x), \xi) .
$$

Hence, when $\eta \rightarrow 0^{+}$, thanks to Lebesgue dominated convergence theorem the expression in (7.4) tends to

$$
|[\psi](x)|\left(\nu_{x}(\{\alpha(x)\}) g^{\prime}(\alpha(x))+\int_{(\alpha(x), 2 M]} \frac{\partial \varphi}{\partial y}(\alpha(x), \xi) \mathrm{d} \nu_{x}(\xi)\right) .
$$


In case $[u](x)[\psi](x)<0$, since $|[u](x)+\eta[\psi](x)|<|[u](x)|=\alpha(x)$ we have

$$
\begin{aligned}
\int_{[0,2 M]} \frac{\varphi(|[u](x)+\eta[\psi](x)|, \xi \vee(|[u](x)+\eta[\psi](x)|))-\varphi(\alpha(x), \xi)}{\eta} \mathrm{d} \nu_{x}(\xi) & =\int_{[0,2 M]} \frac{\varphi(|[u](x)+\eta[\psi](x)|, \xi)-\varphi(\alpha(x), \xi)}{\eta} \mathrm{d} \nu_{x}(\xi) .
\end{aligned}
$$

When $\eta \rightarrow 0^{+}$, using once again Lebesgue dominated convergence theorem, we get that the last expression converges to

$$
[\psi](x) \operatorname{sgn}([u](x)) \int_{[0,2 M]} \frac{\partial \varphi}{\partial y}(\alpha(x), \xi) \mathrm{d} \nu_{x}(\xi)=-|[\psi](x)| \int_{[0,2 M]} \frac{\partial \varphi}{\partial y}(\alpha(x), \xi) \mathrm{d} \nu_{x}(\xi) .
$$

Collecting (7.5) and (7.6) and applying Lebesgue dominated convergence theorem we get the last two lines of relation (7.1).

In the next proposition we give an equivalent formulation for the Euler-Lagrange conditions.

Proposition 7.2. Let $t \in[0, T]$ be fixed and let $(u, \nu) \in a(t, w)$. Then (7.1) holds if and only if the following two conditions are fulfilled:

(a) u satisfies

$$
\begin{cases}\Delta u=0 & \text { in } \mathcal{D}^{\prime}(\Omega \backslash \Gamma), \\ u=w(t) & \text { on } H^{\frac{1}{2}}\left(\partial_{D} \Omega\right), \\ \partial_{\mathbf{n}} u=0 & \text { on } H^{-\frac{1}{2}}\left(\partial \Omega \backslash \partial_{D} \Omega\right), \\ \partial_{\mathbf{n}} u^{+}=\partial_{\mathbf{n}} u^{-} & \text {on } H^{-\frac{1}{2}}(\Gamma) ;\end{cases}
$$

(b) there exists $h \in L^{\infty}(\Gamma)$ such that

$$
\begin{cases}\left\langle\partial_{\mathbf{n}} u,[\psi]\right\rangle=\int_{\Gamma} h[\psi] \mathrm{d} \mathcal{H}^{N-1} & \forall \psi \in H_{0}^{1}\left(\Omega \backslash \Gamma, \partial_{D} \Omega\right), \\ |h| \leq \sigma \nu_{x}(\{0\})+\int_{(0,2 M]} \frac{\partial \varphi}{\partial y}\left(0^{+}, \xi\right) \mathrm{d} \nu_{x}(\xi) & \mathcal{H}^{N-1} \text {-a.e. in } J_{u}^{c} \cap\{\alpha=0\}, \\ |h| \leq \int_{[0,2 M]} \frac{\partial \varphi}{\partial y}\left(0^{+}, \xi\right) \mathrm{d} \nu_{x}(\xi) & \mathcal{H}^{N-1} \text {-a.e. in } J_{u}^{c} \cap\{\alpha>0\}, \\ h \operatorname{sgn}[u]=\int_{[0,2 M]} \frac{\partial \varphi}{\partial y}(|[u]|, \xi) \mathrm{d} \nu_{x}(\xi) & \mathcal{H}^{N-1} \text {-a.e. in }\{0<|[u]|<\alpha\}, \\ h \operatorname{sgn}[u] \in \Sigma & \mathcal{H}^{N-1} \text {-a.e. in }\{0<|[u]|=\alpha\},\end{cases}
$$

where $\Sigma$ is the segment

$$
\Sigma:=\left\{\int_{[0,2 M]} \frac{\partial \varphi}{\partial y}(\alpha, \xi) \mathrm{d} \nu_{x}(\xi)+r \nu_{x}(\{\alpha\})\left(g^{\prime}(\alpha)-\frac{\partial \varphi}{\partial y}(\alpha, \alpha)\right): r \in[0,1]\right\} .
$$

Proof. Let us prove the two implications.

\section{Step 1. Show that $(7.1) \Rightarrow$ (a) and (b)}

Specifying (7.1) for an arbitrary $\psi \in H_{0}^{1}\left(\Omega, \partial_{D} \Omega\right)$ we obtain relations $(7.7)_{1}-(7.7)_{4}$. At this point, integrating by parts we have

$$
\int_{\Omega \backslash \Gamma} \nabla u \cdot \nabla \psi \mathrm{d} x=-\left\langle\partial_{\mathbf{n}} u,[\psi]\right\rangle
$$


for every $\psi \in H_{0}^{1}\left(\Omega \backslash \Gamma, \partial_{D} \Omega\right)$. Arguing in the same way as in [2], we obtain the existence of a function $h \in L^{\infty}(\Gamma)$ such that $(7.8)_{1}$ holds. From (7.1), using $(7.9)$ and $(7.8)_{1}$ and since $H^{\frac{1}{2}}(\Gamma)$ is dense in $L^{1}(\Gamma)$ we get that

$$
\begin{aligned}
-\int_{\Gamma} h z \mathrm{~d} \mathcal{H}^{N-1} & +\int_{\Gamma}|z| 1_{\{0=|[u]|=\alpha\}}\left(\sigma \nu_{x}(\{0\})+\int_{(0,2 M]} \frac{\partial \varphi}{\partial y}\left(0^{+}, \xi\right) \mathrm{d} \nu_{x}(\xi)\right) \mathrm{d} \mathcal{H}^{N-1} \\
& +\int_{\Gamma}|z| 1_{\{0=|[u]|<\alpha\}} \int_{[0,2 M]} \frac{\partial \varphi}{\partial y}\left(0^{+}, \xi\right) \mathrm{d} \nu_{x}(\xi) \mathrm{d} \mathcal{H}^{N-1} \\
& +\int_{\Gamma} z \operatorname{sgn}[u] 1_{\{0<|[u]|<\alpha\}} \int_{[0,2 M]} \frac{\partial \varphi}{\partial y}(|[u]|, \xi) \mathrm{d} \nu_{x}(\xi) \mathrm{d} \mathcal{H}^{N-1} \\
& +\int_{\Gamma}|z| 1_{\{0<[u] z=\alpha|z|\}}\left(g^{\prime}(\alpha) \nu_{x}(\{\alpha\})+\int_{(\alpha, 2 M]} \frac{\partial \varphi}{\partial y}(\alpha, \xi) \mathrm{d} \nu_{x}(\xi)\right) \mathrm{d} \mathcal{H}^{N-1} \\
& -\int_{\Gamma}|z| 1_{\{0>[u] z=-\alpha|z|\}} \int_{[0,2 M]} \frac{\partial \varphi}{\partial y}(\alpha, \xi) \mathrm{d} \nu_{x}(\xi) \mathrm{d} \mathcal{H}^{N-1} \geq 0
\end{aligned}
$$

for every $z \in L^{1}(\Gamma)$. Evaluating the last relation first for functions $z \geq 0$ and then for functions $z \leq 0$ arbitrary, by a localization argument we obtain (7.8).

Step 2. Show that $(a)$ and $(b) \Rightarrow(7.1)$

Conversely, applying (7.7) $)_{1}$ to an arbitrary $\psi \in H_{0}^{1}\left(\Omega \backslash \Gamma, \partial_{D} \Omega\right)$, integrating by parts and using conditions (a) and (b) we get (7.1).

Acknowledgements. The authors would like to thank Gianni Dal Maso and Massimiliano Morini for many useful and interesting discussions. This work is part of the Project "Calculus of Variations" 2004, supported by the Italian Ministry of Education, University, and Research.

\section{REFERENCES}

[1] G.I. Barenblatt, The mathematical theory of equilibrium cracks in brittle fracture. Adv. Appl. Mech. 7 (1962) 55-129.

[2] F. Cagnetti, A vanishing viscosity approach to fracture growth in a cohesive zone model with prescribed crack path. Math. Models Methods Appl. Sci. 18 (2008) 1027-1071.

[3] D.L. Cohn, Measure theory. Reprint of the 1980 original, Birkhäuser, Boston, USA (1993).

[4] G. Dal Maso and R. Toader, A model for the quasi-static growth of brittle fractures: existence and approximation results. Arch. Ration. Mech. Anal. 162 (2002) 101-135.

[5] G. Dal Maso and C. Zanini, Quasi-static crack growth for a cohesive zone model with prescribed crack path. Proc. Roy. Soc. Edinburgh Sect. A 137 (2007) 253-279.

[6] G. Dal Maso, G.A. Francfort and R. Toader, Quasistatic crack growth in nonlinear elasticity. Arch. Ration. Mech. Anal. 176 (2005) 165-225.

[7] G.A. Francfort and J.-J. Marigo, Revisiting brittle fracture as an energy minimization problem. J. Mech. Phys. Solids 46 (1998) 1319-1342.

[8] A. Mielke, Evolution of rate-independent systems, in Handbook of differential equations, evolutionary equations 2, C.M. Dafermos and E. Feireisl Eds., Elsevier, Amsterdam, The Netherlands (2005) 461-559.

[9] J. Neveu, Discrete-Parameter Martingales. American Elsevier, Amsterdam, The Netherlands (1975).

[10] M. Valadier, Young measures, in Methods of nonconvex analysis (Varenna, 1989) 1446, Lect. Notes Math., Springer, Berlin, Germany (1990) 152-188. 